\title{
Continuous Polling Models and Application to Ferry Assisted WLAN
}

\author{
Veeraruna Kavitha • Eitan Altman
}

Received: date / Accepted: date

\begin{abstract}
In this paper we focus on a class of polling systems encountered while modeling the ferry based wireless local area network (FWLAN). A moving ferry, while walking in a predetermined cyclic path, communicates with the static nodes (or users) of the network via a wireless link. The ferry is assumed to stop and communicate with a node that has a packet to send or to receive, when it is closest to that node. The location distribution of the node to which or from which a packet arrives is assumed to have a support of positive Lebesgue measure. These features imply that polling models with finite number of queues cannot be used to model the system. We study in this paper the continuous polling systems with service disciplines that model the use of the FWLAN (and that are more complex than the classical exhaustive or gated services). Our approach is based on discretization of the continuous polling model. We propose a special way of discretizing the continuous system such that: 1) the known Pseudo conservation laws can be applied to obtain the stationary expected workload of the discrete systems; 2) the limit, of these 'discretized' expected workloads, equals the stationary expected workload of the continuous system. Our results rely heavily on fixed point analysis of infinite dimensional operators.
\end{abstract}

\section{Introduction}

Polling systems are a class of queueing systems wherein a single server attends to a number of queues. Systems with a finite number of queues (called discrete polling systems) have been analyzed to a good extent and there is good understanding of their

This work was sponsored by The Indo-French Center for the Promotion of Advanced Research under CEFIPRA Project\# 4000-IT-1

Veeraruna Kavitha

INRIA, Sophia Antipolis and LIA, University of Avignon, France

E-mail: Kavitha.Voleti_Veeraruna@sophia.inria.fr

Eitan Altman

INRIA, Sophia Antipolis

E-mail: Eitan.Altman@sophia.inria.fr 
stability conditions and on the Pseudo conservation laws for a variety of discrete polling systems in $[17,5,3,4,22,6]$ etc.

Continuous polling systems are the ones in which the location of arrivals as well as of the service can occur anywhere in a continuum of locations. Continuous polling were first introduced by Fuhrmann and Cooper [11], further studied, explored by Coffman and Gilbert $[7,8]$ and Kroese and Schmidt $[16,13-15]$ in a series of works. Stability of such polling systems is discussed in $[21,12,20,18]$.

It is relatively more complicated to study continuous systems and these are usually analyzed under simplified conditions, which we refer as 'symmetric conditions': every arrival picks up an uniformly-distributed landing site on the circle, settles down there and awaits service while the server is moving at a constant speed and in a fixed direction around the circle. Further the service time requirements of the users do not depend upon the position of the user.

The work on snowplowing systems in $[9,10]$ generalizes many of the above assumptions and studies a more general continuous system. For example, the incoming work-flow to the system is taken to be a general Levy random measure and the walking times are assumed to be random.

In previous literature so far, the continuous polling systems have been mostly analyzed with standard gated/exhaustive service. In such systems, the server, while moving in a cyclic path, attends the users waiting anywhere on its path the first time it encounters them. But there are other important service disciplines (e.g., globally gated, elevator service etc) which are studied in a discrete polling system. In a globally gated service discipline ([5]), the server attends the users the first time it encounters them after they are tagged and all the users are tagged whenever the ferry reaches a global point. Elevator service ([19]) is similar to globally gated service except that the server reverses its direction at the end of every cycle. There can be applications, wherein the users need to be supported via a mixture of the above service disciplines in a continuous polling system. For example, in a Ferry assisted Wireless LAN all uplink transmissions (data uploaded to BS via the Ferry from static sources) use an exhaustive discipline while all downlink transmissions (data downloaded to a sink from the Ferry which had earlier received the same data from BS) have to be modeled using a globally gated or an elevator regime.

The central idea of this paper is to use the known Pseudo conservation laws (known for a discrete system) for obtaining the stationary expected virtual workload of a continuous system (which supports mixture of services) via discretization. We discretize the continuous polling system in a special way such that the known Pseudo conservation laws can be applied for the resulting discrete systems and the limit, of these 'discrete' stationary workloads, equal that of the continuous system. We achieved this by utilizing the fixed point equations that can result because of stationarity. We basically utilize the periodic behavior in the system, identify certain quantities whose moments must be equal at the beginning and at the end of a cycle because of stationarity (stationarity implies palm stationarity) and hence obtain fixed point equations for the stationary moments. We identify those quantities, which can further be utilized for expressing the stationary expected workload in the system. We obtained the required limit majorly via continuous parameter (the number of discretization levels is the parameter here) dependence of the fixed points. Further, we obtain an expression for the expected stationary virtual workload abandoning the standard symmetric assumptions. Discretization approach is used for mixed service systems, while, the stationary workload for Globally gated and elevator service disciplines is obtained directly. 
In this paper, incoming jobs arrive to the system following a Poisson process. Our approach can be used for studying other types of continuous polling whenever one can solve the related discrete problem and use similar limiting arguments. In [28] we used the same approach to study a continuous system with rerouting.

The second part of the paper applies the Polling system results to study a Ferry assisted Wireless Local Area Network (FWLAN). Message Ferry is a mobile relay station that serves as "postman" to deliver (collect) messages to (from) the static or dynamic wireless nodes in a sparse network, where direct connectivity is not possible. Mobile BS have been proposed in the context of Mobile Ad Hoc Networks [27], Vehicular Ad-Hoc Networks (Vanets) [25] and in Wireless (static) Sensor Networks [26]. In the UmassDiesel project, computers have been installed in 30 out of 40 buses and these then serve as Message Ferry to deliver messages to throw boxes ([23]). In a related paper [27], the message ferry serves some fixed finite number of nodes and the routes are designed based on the mobility models of those finite number of nodes. In contrast, in our work the ferry serves all the nodes that arrive anywhere in the given area as and when they arrive and the optimal routes are designed based on the arrival process, service requirements, network architecture, radio conditions, etc.

The ferry moves periodically in the cyclic route and either halts at predetermined finite number of stops or halts on its way only when it encounters a user with a request. We refer the former as BUS mode while the later one as TAXI mode. BUS mode has been studied using discrete polling systems in [30], while this paper focuses on TAXI mode, using the continuous polling results. In the TAXI mode, every point on the ferry path is a potential stop and each stop is assigned an area that contains all points closer to that stop than to other stops. Upon reaching a stop, the ferry collects (uplink) and dumps (downlink) data from or to the area assigned to it.

An important performance measure for FWLAN design is the expected waiting time, i.e., the average time an arrival has to wait before it's service starts. The waiting time depends on: 1) the over-all service times (the time required to complete an uplink or downlink request using wireless medium); 2) the walking time, i.e., the time taken by the ferry to traverse the cyclic path once. The over-all service times reduce if one uses longer ferry routes as, then, the users can be served by the ferry standing relatively at a shorter distance. However the walking time increases with longer routes. The objective of this paper is to study these trade-offs using stationary expected workload performance. By this, we minimize the expected waiting times at all users in the Pareto sense.

One of the important contributions of this paper is to show how theory of polling systems can be mapped to obtain interesting performance measures for some interesting configurations of FWLAN. These performance measures are used for designing optimal ferry routes (in a given class of routes) or for designing optimal number of stops (for a given type of ferry route ([30])) and or for designing the location of base station(s). However one can think of many more applications of this mapping. For example, in [24] the authors consider a wireless scenario with many ferries and user groups. Their aim was to group ferries and users into coalitions in a optimal way. Each coalition in their paper was a simple example of ferry based wireless LAN. Our results can probably be used to form these optimal coalitions for more complicated architectures.

The continuous polling models are studied in section 2 while ferry based wireless LAN examples are analyzed in sections 3 and 4 . Some proofs are available in Appendices. 


\section{Continuous Polling Systems}

In a continuous polling system, the server moves continuously and stops at point $q$ only when it finds a user with a request. The server is moving continuously on a circle $\mathcal{C}$ of radius $\gamma$ with speed $\alpha$. The arrival process is modeled by a Poisson process with intensity $\lambda$ and every arrival is associated with two marks, the position $Q \in \mathcal{C}$ distributed as $P_{Q}$ and the service times $B$. The service times in general can depend upon the position $Q$ of the arrival. Let $b(q) b^{(2)}(q)$ represent the conditional first two moments of the service time $B$ conditioned on the event that the position of arrival is at point $q$. However the service times of different users are independent of each other.

The server either provides pure or mixed type of service to the users. In a pure globally gated service system, the server closes a fictitious gate each time it arrives at a fixed point in the circle (we refer it as 0 ) and tags all the users that arrived before the closure of the gate. The time period between two consequent arrivals of the server at 0 is referred as cycle. In every cycle, the server moves along the circular path and serves only the tagged users as and when it encounters one. Note here that some times an user may be served only the second time the server encounters it after it's arrival. On the contrary in a pure gated/exhaustive service polling system, the users are served more immediately after their birth: they are served the first time, the server encounters them. In a discrete polling system, there is a difference between gated service (the server closes a local gate at every queue and leaves the queue only after serving the users that appeared before the gate closure) and exhaustive service (the server also attends the users that arrived during the service of already existing users and it leaves the queue only when the queue is empty). But the two converge to the same service in a continuous polling system with $P_{Q}$ continuous: at any queue (which is a point in the continuous case), the probability of a new user appearing at the same point is zero.

In a mixed service polling system, the server attends some of the users with globally gated service and the rest with exhaustive/gated service. Let $p_{g g}, p_{g}=1-p_{g g}$ respectively represent the probability that an arrival is a globally gated user and a gated user. Let $b_{g}(q), b_{g}^{(2)}(q)$ represent the conditional first and second moments of the service time given that the arrival is at point $q$ and further given that the arrival is a gated user. Let $b_{g g}(q), b_{g g}^{(2)}(q)$ represent same quantities for a globally gated user. Let $\bar{b}:=E[b(Q)]$, $\bar{b}_{g g}:=E\left[b_{g g}(Q)\right]$ and $\bar{b}_{g}:=E\left[b_{g}(Q)\right]$ (expectations are with respect to ${ }^{1} P_{Q}$ ) represent the unconditional moments. Note that $\bar{b}=\bar{b}_{g} p_{g}+\bar{b}_{g g} p_{g g}$. Similarly define the second moments, $\bar{b}_{g}^{(2)}:=E\left[b_{g}^{(2)}(Q)\right], \bar{b}_{g g}^{(2)}:=E\left[b_{g g}^{(2)}(Q)\right]$ and $\bar{b}^{(2)}:=E\left[b^{(2)}(Q)\right]$.

Notations : The variables like $b, b_{g}, b_{g g}, f_{Q}, \tau, v$ represent the functions on interval $[0,|\mathcal{C}|]$ while terms like $b(q)$ or $\tau(q)$ represent the function values at a point $q \in[0,|\mathcal{C}|]$. The bar of the same variable, like $\bar{b}_{g}$ represent the average w.r.t. to the position distribution $P_{Q}$, i.e., $\bar{b}_{g}=E\left[b_{g}(Q)\right]$. Similarly variables like $\tau^{(2)}, \tilde{\tau}^{(2)}$ etc. are functions on $[0,|\mathcal{C}|] \times[0,|\mathcal{C}|]$ while $\tau^{(2)}\left(q, q^{\prime}\right)$ represents the function value at $\left(q, q^{\prime}\right)$. Let $q \wedge|\mathcal{C}|$ represent the minimum value, $\min \{q,|\mathcal{C}|\}$. The expectation with respect to the stationary measure of the process under consideration is given by $E$ while $E^{0}$ represents the expectation with respect to Palm stationary measure.

Virtual workload of a polling system is defined as the total workload corresponding to all the waiting users, i.e., the sum of the service times of all the waiting users. It is an important performance measure and will be used in later sections for analyzing

1 Right now the position of arrival is distributed the same way for gated as well as globally gated users. But the results can easily be generalized to separate $P_{Q, g}$ and $P_{Q, g g}$. 
interesting application in delay tolerant networks. Not much theory is available for calculating the expected virtual workload of polling systems with arrivals in a continuum. In this section we derive new (stationary expected workload) results related to continuous polling systems. Throughout we consider stationary and ergodic systems. An exhaustive/gated continuous polling system is analyzed in [16] under symmetric conditions and we summarize the same in subsection 2.1. The main contribution of this paper towards polling systems are available in subsections 2.2, 2.3 and 2.4 in the form of stationary expected virtual workload derivations for polling system respectively with a globally gated service, elevator and mixed service. These results also cover the gated/exhaustive service system under asymmetric conditions.

\subsection{Exhaustive/Gated Service}

An exhaustive service continuous polling system under symmetric conditions is analyzed in [16]. They have many more results but we only present the ones relevant to this paper, i.e., the expression for expected virtual workload under stationarity. The users arrive uniformly on the circumference with $|\mathcal{C}|=1$ as a Poisson process with intensity $\lambda$, i.e., $P_{Q}$ is uniform. Further the service time moments $b_{g}(q), b_{g}^{(2)}(q)$ are assumed to be same at all points $q$ and are equal to $\bar{b}_{g}$ and $\bar{b}_{g}^{(2)}$ respectively. Then the stationary expected number of customers on the circle is given by [16, Theorem 5.1]

$$
E[N]_{g}=\lambda \bar{b}_{g}+\frac{\lambda\left(\alpha^{-1}+\lambda \bar{b}_{g}^{(2)}\right)}{2\left(1-\lambda \bar{b}_{g}\right)} .
$$

In this case they also considered the user under service. Excluding the user under service, i.e., the stationary expected number of waiting customers equal:

$$
E[N]_{g}^{w}=\frac{\lambda\left(\alpha^{-1}+\lambda \bar{b}_{g}^{(2)}\right)}{2\left(1-\lambda \bar{b}_{g}\right)} .
$$

From (1), by Wald's lemma the virtual workload, the workload due to the waiting customers, equals:

$$
V_{g}^{s y m}:=\bar{b}_{g} E[N]_{g}^{w}=\frac{\lambda \bar{b}_{g}\left(\alpha^{-1}+\lambda \bar{b}_{g}^{(2)}\right)}{2\left(1-\lambda \bar{b}_{g}\right)}
$$

\subsection{Globally Gated Service}

In this subsection we derive the corresponding results for a globally gated service. Further we don't assume symmetric conditions and work under more general conditions as explained before. We map the circumference to a line of length $|\mathcal{C}|:=2 \pi \gamma$ with the starting point (the point at which the global gate is closed) mapped to 0. 
2.2.1 Computation of the moments of the Cycle time in stationary regime:

Let $C_{n}$ represent the time taken to complete the $n^{\text {th }}$ cycle. Let $\mathcal{N}(C)$ represent the number of Poisson arrivals in time $C$. With $B_{i}^{n}$ representing the service time of the $i^{\text {th }}$ user arrived during the $n-1^{\text {th }}$ cycle, the adjacent cycles times can be related using

$$
C_{n}=\sum_{i}^{\mathcal{N}\left(C_{n-1}\right)} B_{i}^{n}+|\mathcal{C}| \alpha^{-1}
$$

By first conditioning on $\mathcal{N}\left(C_{n-1}\right)$ and then on $C_{n-1}$, it is easy to see that,

$$
E\left[\sum_{i}^{\mathcal{N}\left(C_{n-1}\right)} B_{i}^{n}\right]=\lambda E\left[C_{n-1}\right] \int_{0}^{|\mathcal{C}|} b_{g g}(q) P_{Q}(d q) .
$$

Thus under (Palm) stationarity ${ }^{2}$,

$$
c_{*}:=E^{0}\left[C_{n}\right]=\frac{|\mathcal{C}| \alpha^{-1}}{1-\lambda \bar{b}_{g g}} .
$$

From the above it is clear that the system is stable only if the factor $\rho:=\lambda \bar{b}_{g g}$ is strictly less than 1 . This is the usual condition for stability and throughout this paper we assume stability. In a similar way as,

$$
\left(\sum_{i}^{\mathcal{N}\left(C_{n-1}\right)} B_{i}^{n}\right)^{2}=\sum_{i}^{\mathcal{N}\left(C_{n-1}\right)}\left(B_{i}^{n}\right)^{2}+\sum_{i \neq j} B_{i}^{n} B_{j}^{n}
$$

and because $E\left[\mathcal{N}\left(C_{n}\right)^{2}-\mathcal{N}\left(C_{n}\right)\right]=\lambda^{2} E^{0}\left[C_{n}^{2}\right]\left(\right.$ with $\left.c_{*}^{(2)}:=E^{0}\left[C_{n}^{2}\right]\right)$,

$$
c_{*}^{(2)}=\lambda c_{*} \bar{b}_{g g}^{(2)}+\lambda^{2} c_{*}^{(2)} \bar{b}_{g g}^{2}+|\mathcal{C}|^{2} \alpha^{-2}+2|\mathcal{C}| \alpha^{-1} \lambda c_{*} \bar{b}_{g g} .
$$

Thus,

$$
c_{*}^{(2)}=\frac{|\mathcal{C}| c_{1}^{-1}}{1-\lambda \bar{b}_{g g}} \frac{|\mathcal{C}| \alpha^{-1}+\lambda \bar{b}_{g g}^{(2)}+|\mathcal{C}| \alpha^{-1} \lambda \bar{b}_{g g}}{1-\lambda^{2} \bar{b}_{g g}^{2}} .
$$

2 Let $\phi_{n}$ represent the time at which the server visits the global gate, 0 , in the $n^{\text {th }}$ cycle. Then $C_{n}=\phi_{n}-\phi_{n-1}$. For any stationary point process, for example in our case $\left\{\phi_{n}\right\}$, there will be two associated probabilities: Stationary and Palm Stationary ([1]). In general, $\left\{\phi_{n}\right\}$ are defined such that $\phi_{0} \leq 0<\phi_{1}$. Palm probabilities are the stationary probabilities obtained after conditioning on the event that $\left\{\phi_{0}=0\right\}$ (see [1]). Throughout the paper, the expectation under Palm stationary measure is represented by $E^{0}$ and the corresponding moments are usually denoted with a $*$ as sub-script. In [1], the stationary moment of the residual cycle $C_{1}$ (which we refer as $C_{R}$ ) as well as the past cycle $C_{0}$ (which we refer as $C_{P}$ ) is obtained in terms of Palm probabilities as

$$
E\left[C_{R}\right]=E\left[C_{P}\right]=\frac{E^{0}\left[C_{1}^{2}\right]}{2 E^{0}\left[C_{1}\right]} .
$$

This result is also explicitly derived specifically for cycle times in a special polling system in Section 3.1 of [2]. This result is used throughout. 
2.2.2 Stationary Expected Virtual Workload

An user arriving at $q$ and at an arbitrary time epoch (which is modeled as occurred at time 0 ) has to wait, for his service to start, on average for a time equal to the sum of three components : 1) the average residual cycle time of the ongoing cycle $C_{R} ; 2$ ) the time taken by the server to reach the point $q$ from $\left.0, q \alpha^{-1} ; 3\right)$ the average time taken by the server to attend the users belong to the segment $[0, q]$ that arrived in the entire ongoing cycle $C_{R}+C_{P}$ ( $C_{P}$ represents the past cycle time of the ongoing cycle), $\sum_{i}^{\mathcal{N}\left(C_{P}+C_{R}\right)} B_{i} 1_{\left\{q_{i} \in[0, q]\right\}}$. Thus the stationary expected waiting time of a user given that the user arrives at point $q$ equals (see footnote 2 ),

$$
\begin{aligned}
E[W](q) & =E\left[C_{R}\right]+q \alpha^{-1}+\lambda P_{Q}(Q \in[0, q]) E\left[C_{R}+C_{P}\right] E[B \mid Q \in[0, q]] \\
& =\frac{c_{*}^{(2)}}{2 c_{*}}+q \alpha^{-1}+\lambda \frac{c_{*}^{(2)}}{c_{*}} \widehat{b}_{g g}(q) \text { where } \\
\widehat{b}_{g g}(q) & :=\int_{0}^{q} b_{g g}(y) P_{Q}(d y) .
\end{aligned}
$$

By Little's law ([31]) the stationary expected number of waiting users that belong to infinitesimal segment $[q-d q, q+d q]$ equals,

$$
E[N](d q)=\lambda E[W](q) P_{Q}(d q)
$$

and thus by Wald's Lemma, the stationary expected virtual workload (by virtue of independence) due to users that belong to infinitesimal segment $[q-d q, q+d q]$ equals

$$
V_{g g}(d q)=b_{g g}(q) \lambda E[W](q) P_{Q}(d q) .
$$

Hence the stationary expected virtual workload in the entire system equals

$$
\begin{aligned}
V_{g g} & =\lambda \int_{0}^{|\mathcal{C}|} E[W](q) b_{g g}(q) P_{Q}(d q) \\
& =\lambda\left(\frac{c_{*}^{(2)}}{c_{*}}\left(\frac{\bar{b}_{g g}}{2}+\lambda E\left[\widehat{b}_{g g}(Q) b_{g g}(Q)\right]\right)+\alpha^{-1} E\left[Q b_{g g}(Q)\right]\right) .
\end{aligned}
$$

By interchanging the order of the two integrals in $E\left[\widehat{b}_{g g}(Q) b_{g g}(Q)\right]$,

$$
\begin{aligned}
\int_{0}^{|\mathcal{C}|} \int_{0}^{q^{\prime}} b_{g g}(q) P_{Q}(d q) b_{g g}\left(q^{\prime}\right) & P_{Q}\left(d q^{\prime}\right)=\int_{0}^{|\mathcal{C}|} \int_{q}^{|\mathcal{C}|} b_{g g}\left(q^{\prime}\right) P_{Q}\left(d q^{\prime}\right) b_{g g}(q) P_{Q}(d q) \\
& =\int_{0}^{|\mathcal{C}|}\left(\bar{b}_{g g}-\int_{0}^{q} b_{g g}\left(q^{\prime}\right) P_{Q}\left(d q^{\prime}\right)\right) b_{g g}(q) P_{Q}(d q) \\
& =\bar{b}_{g g}^{2}-\int_{0}^{|\mathcal{C}|} \int_{0}^{q} b_{g g}\left(q^{\prime}\right) P_{Q}\left(d q^{\prime}\right) b_{g g}(q) P_{Q}(d q)
\end{aligned}
$$

and so, $E\left[b_{g g}(Q) \widehat{b}_{g g}(Q)\right]=\bar{b}_{g g}^{2} / 2$. From $(3)$, (4) the virtual workload simplifies to:

$$
V_{g g}=\lambda\left(\frac{|\mathcal{C}| \alpha^{-1}\left(1+\lambda \bar{b}_{g g}\right)+\lambda \bar{b}_{g g}^{(2)}}{1-\lambda \bar{b}_{g g}} \frac{\bar{b}_{g g}}{2}+\alpha^{-1} E\left[Q b_{g g}(Q)\right]\right) .
$$


Symmetric Case: We specialize to the symmetric case where $b(q) \equiv b, b^{(2)}(q) \equiv b^{(2)}$

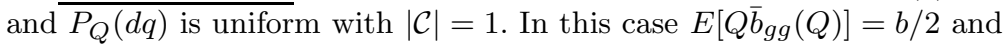

$$
V_{g g}^{s y m}=\lambda \bar{b}_{g g}\left(\frac{\lambda \bar{b}_{g g}^{(2)}}{2\left(1-\lambda \bar{b}_{g g}\right)}+\frac{\alpha^{-1}}{1-\lambda \bar{b}_{g g}}\right) .
$$

\subsection{Elevator Service}

This polling system is similar to that in subsection 2.2 except that the server moves in the opposite direction every time it reaches the point at which the global gate closes. That is, the server moves in counter clockwise direction in alternate, say odd numbered cycles and in the reverse direction in the even numbered cycles. In [19], authors describe the scheme for discrete polling system. The analysis of the continuous counterpart will be similar to that in 2.2 and we mention only the points of difference.

Stationary moments of the Cycle time: The adjacent cycles times can once again be related using

$$
C_{n}=\sum_{i}^{\mathcal{N}\left(C_{n-1}\right)} B_{i}^{n}+|\mathcal{C}| \alpha^{-1} \text { and } C_{n-1}=\sum_{i}^{\mathcal{N}\left(C_{n-2}\right)} B_{i}^{n-1}+|\mathcal{C}| \alpha^{-1}
$$

Under stationarity $C_{n}$ will be distributed same as $C_{n-2}$ and $C_{n-1}$ as $C_{n-3}$. Because of the IID nature of the services times $\left\{B_{i}^{n}\right\}$ across any cycle, for the above relations to hold $C_{n}$ should be distributed same as $C_{n-1}$. Thus again under stationarity,

$$
\begin{aligned}
c_{*} & =\frac{|\mathcal{C}| \alpha^{-1}}{1-\lambda \bar{b}_{g g}} \\
c_{*}^{(2)} & =\frac{|\mathcal{C}| c_{1}^{-1}}{1-\lambda \bar{b}_{g g}} \frac{|\mathcal{C}| \alpha^{-1}+\lambda \bar{b}_{g g}^{(2)}+|\mathcal{C}| \alpha^{-1} \lambda \bar{b}_{g g}}{1-\lambda^{2} \bar{b}_{g g}^{2}} .
\end{aligned}
$$

Expected waiting time for a random user : Here again the time the user arriving at $q$ has to wait for his service will be equal to the sum of three components as in subsection 2.2 but the server will be moving either in clockwise direction or in anticlockwise direction with half probability. Thus the expected waiting time at any point $q$ equals :

$$
\begin{aligned}
E[W](q) & =E\left[C_{R}\right]+\frac{1}{2}\left(q \alpha^{-1}+\lambda P_{Q}(Q \in[0, q]) E\left[C_{R}+C_{P}\right] E[B \mid Q \in[0, q]]\right) \\
+ & \frac{1}{2}\left((|\mathcal{C}|-q) \alpha^{-1}+\lambda P_{Q}(Q \in[q,|\mathcal{C}|]) E\left[C_{R}+C_{P}\right] E[B \mid Q \in[q,|\mathcal{C}|]]\right) \\
& =\frac{c_{*}^{(2)}}{2 c_{*}}+\frac{|\mathcal{C}| \alpha^{-1}}{2}+\lambda \frac{c_{*}^{(2)}}{2 c_{*}} \bar{b}_{g g}=\frac{\lambda \bar{b}_{g g}^{(2)}}{2\left(1-\lambda \bar{b}_{g g}\right)}+|\mathcal{C}| \alpha^{-1}
\end{aligned}
$$

which is independent of the position $q$. As anticipated, the expected waiting times are independent of the position at which the user waits. The stationary expected workload can be derived as before and equals

$$
V_{\text {elevator }}=\lambda \bar{b}_{g g}\left(\frac{\lambda \bar{b}_{g g}^{(2)}}{2\left(1-\lambda \bar{b}_{g g}\right)}+|\mathcal{C}| \alpha^{-1}\right) .
$$




\subsection{Mixed Service}

This is a system in which the server attends some of the users with globally gated service and the rest with exhaustive service. For users with globally gated service the server closes the gate when it touches upon 0 and serves in the current cycle only those which arrived before the gate closure as in subsection 2.2. The rest of the users will be served, immediately, as in subsection 2.1. We obtain the stationary expected virtual workload of the mixed system as a limit of the same for a 'certain' set of the discrete polling systems, under an extra assumption: the service times are bounded with probability one by a constant that is less than infinity.

Theorem 1 Assume $P_{Q}(d q)=f_{Q}(q) d q$, i.e., $f_{Q}$ is the density of $P_{Q}$. Assume further that $b_{g}, b_{g g}$ and $f_{Q}$ are continuous. Also, there exists a $\hat{B}<\infty$ such that, the service times (irrespective of the position of the arrival) are bounded by $\hat{B}$ with probability one, i.e., $P(B<\hat{B})=1$. Then there exists a threshold $\hat{\mathbf{B}}$ (discussed in the proof) and for all service times with upper bound $\hat{B} \leq \hat{\mathbf{B}}$, the expected stationary virtual workload for the mixed service continuous polling system, $V_{m i x}$, is given by the limit of the corresponding one, $V_{m i x}^{\sigma}$, for a "certain" discrete polling system with $2 \sigma$ number of queues ${ }^{3}$ :

$$
\begin{aligned}
V_{\text {mix }}=\lim _{\sigma \rightarrow \infty} V_{\text {mix }}^{\sigma}= & \lambda \bar{b} \frac{\lambda \bar{b}^{(2)}}{2(1-\lambda \bar{b})}+p_{g g} \lambda \alpha^{-1} E\left[Q b_{g g}(Q)\right]+\frac{\lambda \bar{b}|\mathcal{C}| \alpha^{-1}}{2} \\
& +\frac{\lambda^{2}|\mathcal{C}| \alpha^{-1}}{2(1-\lambda \bar{b})}\left(\bar{b}^{2}+\left(p_{g g} \bar{b}_{g g}\right)^{2}+p_{g g} p_{g} \bar{b}_{g g} \overline{\widehat{b}}_{g}\right)
\end{aligned}
$$

where $|\mathcal{C}|=2 \pi \gamma, \widehat{b}_{g}(q):=E\left[b_{g}(Q) 1_{\{Q \leq q\}}\right]$ and $\widehat{\widehat{b}}_{g}:=E\left[\widehat{b}_{g}(Q)\right]$.

Before we proceed with proving the above theorem we compare the formula (7) with the special cases considered so far. We note that, the general formula (7) matches with (2) of subsection 2.1, when specialized to the symmetric conditions and to the pure gated service (i.e., with $p_{g}=1=1-p_{g g}$ ). It also matches with with globally gated workload (5) derived in subsection 2.2 , when specialized to this pure service (i.e., $\left.p_{g}=0=1-p_{g g}\right)$.

The proof of Theorem 1 is obtained using the following 3 major steps:

1)Discretization: Continuous polling system is converted to an appropriate discrete polling system in subsection 2.4.1, for which the Pseudo conservation laws and hence the expected virtual workload is known (see $[5,3]$ etc.).

Let $\delta^{\sigma}(q)$ for every point $q$ on $\mathcal{C}$ represent the point, in the discrete system with $\sigma$ discretization levels, standing at which the server attends the possible users of point $q$. Let $\delta^{\infty}$ represent the same for continuous system. Note that $\delta^{\infty}(q)=q$ for all $q$, i.e., that $\delta^{\infty}$ is the identity map.

2)Fixed point equations: We express the stationary moments of the time to reach

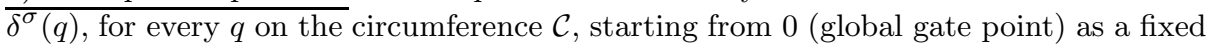
point (in the space of left continuous and right limit functions) of an affine linear operator in subsection 2.4.2. We obtain a common operator $(\mathcal{F}$ defined in subsection 2.4.2), which is further parametrized by a map, $\delta:[0,|\mathcal{C}|] \rightarrow[0,|\mathcal{C}|]$. The fixed point of the common operator at $\delta=\delta^{\sigma}$ gives the required stationary first moments for the discrete

3 The formula (7) was conjectured in [29] itself. However its proof is derived in this paper. Further, we note that there is a small error in the formula published in [29] (in the last term $\widehat{b}_{g}$ is wrongly misplaced with $\bar{b}_{g}$ ) and is corrected here. 
system while that at $\delta=\delta^{\infty}$ (the identity map) corresponds to the continuous system. We show the continuity of these fixed points with respect to $\delta$ via contraction mapping theorem and hence show that the stationary moments of the discrete system converge to that of the continuous system. We obtain the convergence of second moments using similar but more complicated logic.

3)Alternate expression for Virtual Workload: We express the expected virtual workload in terms of the stationary moments of the step (2). Note, that this common expression (given by (19) in subsection 2.4.3) cannot be computed easily and is used only for the proof. Using this common expression, we show the convergence of the stationary expected virtual workload of the discrete system to that of the continuous system in subsection 2.4.3.

\subsubsection{Discretization}

For each integer $\sigma$, consider a discrete polling system with $2 \sigma$ queues, half of which experience globally gated service while the rest half experience gated service. The circumference $|\mathcal{C}|$ is divided to $\sigma$ equal segments $\left\{I_{i}\right\}_{i=1}^{\sigma}$ with $I_{1}=[0, \mathcal{C} / \sigma)$. All the users arriving in area $I_{i}$ are treated as though arriving in queue numbered $2 i-1$ (for gated users) or $2 i$ (for globally gated users). Note that the globally gated users are the tagged ones, i.e., the ones that arrived before the server reached 0 in the current cycle. For every $i$, the server stops upon reaching the starting point, $i^{\sigma}:=(i-1)|\mathcal{C}| / \sigma$, of $I_{i}$ and serves the users of $I_{i}$ (that arrived/tagged before the server reached the stop $i^{\sigma}$ ) before moving further. Hence, $\delta^{\sigma}(q)$, the point standing at which the server attends the users at $q$, equals

$$
\delta^{\sigma}(q)= \begin{cases}\sum_{i=1}^{\sigma} i^{\sigma} 1_{\left\{q \in I_{i}\right\}} \text { with } I_{i}:=\left[i^{\sigma},(i+1)^{\sigma}\right), i^{\sigma}:=\frac{(i-1)|\mathcal{C}|}{\sigma} & \text { if } \sigma<\infty \\ & \text { if } \sigma=\infty .\end{cases}
$$

Within a queue, the server attends the users in a special order which we call as arrival position order. In this special order, the users within a queue are served in the order of their distance from the stop $i^{\sigma}$ of the server, i.e., the user at minimum distance is served first. So, the users are almost served in the same order as done in a continuous system. The main difference $\mathrm{b} / \mathrm{w}$ the continuous system and the discretized system is that some of the users are postponed to the next cycle in the discretized system. This is mainly because of the combined effect of discretization and the gated service. But we will see that the effect of the users, whose service is postponed, reduces to zero as $\sigma$ tends to $\infty$.

Define the following terms related to each stop and or each queue with obvious meanings as below:

$$
\begin{aligned}
& b_{2 i-1}:=E\left[B \mid Q \in I_{i}, \text { gated }\right]=\frac{E\left[b_{g}(Q) 1_{\left\{Q \in I_{i}\right\}}\right]}{P_{Q}\left(I_{i}\right)} \quad b_{2 i-1}^{(2)}:=\frac{E\left[b_{g}^{(2)}(Q) 1_{\left\{Q \in I_{i}\right\}}\right]}{P_{Q}\left(I_{i}\right)} \\
& b_{2 i}:=\frac{E\left[b_{g g}(Q) 1_{\left\{Q \in I_{i}\right\}}\right]}{P_{Q}\left(I_{i}\right)} \quad b_{2 i}^{(2)} \quad:=\frac{E\left[b_{g g}^{(2)}(Q) 1_{\left\{Q \in I_{i}\right\}}\right]}{P_{Q}\left(I_{i}\right)} \\
& l_{i} \quad:=P_{Q}\left(I_{i}\right) \quad \lambda_{2 i-1}:=\lambda l_{i} p_{g} \quad \lambda_{2 i} \quad:=\lambda l_{i} p_{g g} \\
& \rho_{i} \quad:=\lambda_{i} b_{i} \quad \text { and } \quad \rho \quad:=\sum_{i}^{2 \sigma} \rho_{i} \text {. }
\end{aligned}
$$

In the above, $\left\{\lambda_{2 i-1}\right\}_{i=1}^{\sigma},\left\{b_{2 i-1}\right\},\left\{b_{2 i-1}^{(2)}\right\}$ respectively represent the arrival rates, the first and the second moments of the service times at the gated queues while $\left\{\lambda_{2 i}\right\}_{i=1}^{\sigma}$, $\left\{b_{2 i}\right\},\left\{b_{2 i}^{(2)}\right\}$ represent the same for globally gated queues. Factor $\rho$ represents the total 
work load in the system, note that $\rho$ is same for all $\sigma$ and equals $\lambda \bar{b}$. The discrete system is stable only when $\rho<1$. The continuous system is assumed to be stable and hence the stationary moments of Theorem 2 (given in later subsections) exist and hence by the same theorem $\rho<1$. Thus the discrete systems are stable for all $\sigma$.

Expected Virtual Workload for discretized polling system:

This type of a mixed service stable discrete polling system is considered in $[5,3,4]$. By Pseudo Conservation Laws of $[4,3]$ the expected stationary virtual workload of the $\sigma$-polling model with mixed (globally gated and gated) services is:

$$
\begin{array}{r}
V_{\text {mix }}^{\sigma}=\rho \frac{\sum_{j=1}^{\sigma} \lambda_{j} b_{j}^{(2)}}{2(1-\rho)}+\frac{\rho s^{(2)}}{2 s^{(1)}}+\frac{s^{(1)}}{2(1-\rho)}\left[\rho^{2}+\sum_{i=1}^{\sigma} \rho_{i}^{2}\right] \\
+\frac{s^{(1)}}{1-\rho} \sum_{i \in g g} \rho_{i} \sum_{j \leq i} \rho_{j}+\sum_{i \in g g} \rho_{i} \sum_{j<i} s_{j} .
\end{array}
$$

In the above, $s^{(1)}, s^{(2)}$ represent the first and the second moment of the total walking time, $\left\{s_{i}\right\}$ represent the first moments of the walking times between consecutive queues and the remaining terms have similar definitions as before. The results of $[5,3,4]$ are valid for any work conserving order at each queue and hence the results are also valid for our arrival position order.

In our case we have a $2 \sigma$ stable polling system with even numbered queues experiencing the globally gated service and the odd numbered queues experiencing the gated service. Further we have fixed walking times between queues, that between the queues of the same stop is zero, $s_{2 i-1}=0$ and $s_{2 i}=|\mathcal{C}| \alpha^{-1} / \sigma$. Thus the expression for expected stationary virtual workload for our discrete polling system is:

$$
\begin{aligned}
V_{\text {mix }}^{\sigma}=\quad \rho \frac{\sum_{j=1}^{2 \sigma} \lambda_{j} b_{j}^{(2)}}{2(1-\rho)}+\frac{\rho|\mathcal{C}| \alpha^{-1}}{2}+\frac{|\mathcal{C}| \alpha^{-1}}{2(1-\rho)}\left[\rho^{2}+\sum_{i=1}^{2 \sigma} \rho_{i}^{2}\right] \\
\quad+\frac{|\mathcal{C}| \alpha^{-1}}{1-\rho} \sum_{i}^{\sigma} \rho_{2 i} \sum_{j \leq 2 i} \rho_{j}+\sum_{i}^{\sigma} \rho_{2 i} \frac{(i-1)|\mathcal{C}| \alpha^{-1}}{\sigma} .
\end{aligned}
$$

We will prove that the limit of the 'discrete' expected stationary virtual workload, $V_{m i x}^{\sigma}$, indeed equals that of the continuous system. This basically forms the proof of the Theorem 1 and this proof is given in the next two subsections. We conclude this subsection by computing the limit of (9) (proof in Appendix A):

Lemma 1 The limit of $V_{m i x}^{\sigma}$ (9) equals $V_{\text {mix }}$ given by (7) of Theorem 1.

\subsubsection{Fixed point equations}

Let $T_{n}^{\sigma}(q)$ represent the time to reach the point, $\delta^{\sigma}(q)$ of (8), starting from the global point 0 , during the $n^{\text {th }}$ cycle. Let $V_{n}^{\sigma}([a, c])$ represent the workload present at the beginning of the $n^{\text {th }}$ cycle in the segment $[a, c] \subset[0,|\mathcal{C}|]$. Let $\mathcal{T}_{g}([a, c], T)\left(\right.$ or $\mathcal{T}_{g g}([a, c], T)$ in case of globally gated system) represent the total workload of the gated (or globally gated) Poisson arrivals that arrived in segment $[a, c] \subset[0,|\mathcal{C}|]$, such that a user at point $q \in[a, c]$ can arrive during a period of time $T(q)$. They are related in a continuous polling system by (i.e., with $\sigma=\infty$ ):

$$
\begin{aligned}
T_{n}^{\sigma}(q) & =q \alpha^{-1}+V_{n}^{\sigma}([0, q))+\mathcal{T}_{g}\left([0, q), T_{n}^{\sigma}\right) \\
V_{n}^{\sigma}([a, c]) & =\mathcal{T}_{g g}\left([a, c], T_{n-1}^{\sigma}(|\mathcal{C}|)\right)+\mathcal{T}_{g}\left([a, c], T_{n-1}^{\sigma}(|\mathcal{C}|)-T_{n-1}^{\sigma}\right) .
\end{aligned}
$$


In the above, the first term of (10) represents the time taken to walk the distance $q$. The second term, $V_{n}^{\sigma}([0, q])$, represents the time taken to complete service of the globally gated and gated users (placed geographically before $q$ ) that arrived before the server reached 0 . The third term represents the time taken to complete service of the gated users (placed before $q$ ) that arrived during the current cycle, before the server reached their respective $\delta^{\sigma}$ points.

In a discrete polling system, $T_{n}^{\sigma}(q)$ is the time till the server attends all the users belonging to the line segments placed before the point $q,\left\{I_{j}\right\}_{j<q \sigma /|\mathcal{C}|}$, and hence is same for all the points of the same line segment and is given by:

$$
T_{n}^{\sigma}(q)=T_{n}^{\sigma}\left(\delta^{\sigma}(q)\right)=\delta^{\sigma}(q) \alpha^{-1}+V_{n}^{\sigma}\left(\left[0, \delta^{\sigma}(q)\right]\right)+\mathcal{T}_{g}\left(\left[0, \delta^{\sigma}(q)\right], T_{n}^{\sigma}\right) .
$$

Note that the same equation at $\delta=\delta^{\infty}$ matches with the continuous time equation (10). The equation (11) remains the same for the discrete system also.

First Moments: By the lemma below, we obtain integral representation of the first moments of the workloads like, $\mathcal{T}_{g}\left([0, q], T_{n}^{\sigma}\right)$ using which we obtain the integral presentation of the first moments of $T_{n}^{\sigma}$ (proof in Appendix B).

Lemma 2 Let $T:[a, c] \mapsto \mathcal{R}^{+}$be either monotone (increasing or decreasing) or constant nonnegative random function on interval $[a, c]$. Assume that for any $q \in[a, c]$, the service times of the new arrivals and the arrival process at around point $q$ is independent of the system evolution before ${ }^{4}$ time $T(q)$. Let $\mathcal{T}([a, c], T)$ represent the total workload of the Poisson arrivals that arrived in segment $[a, c] \subset[0,|\mathcal{C}|]$, such that a user at point $q \in[a, c]$ can arrive during a period of time $T(q)$. Then with $\tau(q):=E[T(q)]$ for any $q$,

$$
E[\mathcal{T}([a, c], T)]=\lambda \int_{a}^{c} b(q) f_{Q}(q) \tau(q) d q
$$

Let $\mathbf{N}:=\{1,2, \cdots, \infty\}$. Let $\tau_{n}^{\sigma}(q):=E\left[T_{n}^{\sigma}(q)\right]$ represent the first moment of $T_{n}^{\sigma}(q)$ when the number of stops equal $\sigma$ and let $\tau_{n}^{\infty}(q)$ represent the same for continuous system. Substituting (11) in (12) and then applying Lemma 2, we get for any $n \in \mathbf{N}$ :

$$
\begin{aligned}
& \tau_{n}^{\sigma}(q):=\delta(q) \alpha^{-1}+\int_{0}^{\delta(q)} v_{n}^{\sigma}(y) d y+\lambda p_{g} \int_{0}^{\delta(q)} b_{g}(y) f_{Q}(y) \tau_{n}^{\sigma}(y) d y \text { where } \\
& v_{n}^{\sigma}(q):=\lambda p_{g g} b_{g g}(q) \tau_{n-1}^{\sigma}(|\mathcal{C}|) f_{Q}(q)+\lambda p_{g} b_{g}(q)\left(\tau_{n-1}^{\sigma}(|\mathcal{C}|)-\tau_{n-1}^{\sigma}(q)\right) f_{Q}(q) .
\end{aligned}
$$

Let $\mathcal{D}$ represent the space of left continuous functions with right limits on $[0,|\mathcal{C}|]$ equipped with supremum norm:

$$
\text { for any } \tau \in \mathcal{D}, \quad\|\tau\|_{\infty}:=\sup _{q \in[0,|\mathcal{C}|]}|\tau(q)| \text {. }
$$

Consider a parametrized function $\mathcal{F}: \mathcal{D} \times \mathcal{D} \mapsto \mathcal{D}$ defined point-wise as below:

$$
\mathcal{F}(\tau ; \delta)(q):=\delta(q) \alpha^{-1}+\lambda \tau(|\mathcal{C}|) \int_{0}^{\delta(q) \wedge|\mathcal{C}|} b(y) f_{Q}(y) d y \quad \text { for all } q \in \mathcal{C} .
$$

4 Note that this assumption is satisfied by our polling systems (discrete as well as continuous ones) because the system evolution is effected by an arrival only after it is served and here $T_{n}^{\sigma}(q)$ (for which this lemma would be applied) represents the time instance at which it starts serving the users of the segment in which $q$ resides. 
Let $\tau_{*}^{\sigma}(q)$, for $\sigma \in \mathbf{N}$, represent the (Palm) stationary moment corresponding to $\tau_{n}^{\sigma}(q)$. By stationarity ${ }^{5}$ from equation (13) the stationary first moments, $\tau_{*}^{\sigma}$, of the discrete system is a fixed point of the parametrized function $\mathcal{F}$, at $\delta^{\sigma}$ with $\sigma<\infty$, while that of the continuous system is fixed point of the same function at $\delta^{\infty}$. Note here that $b(q)=p_{g} b_{g}(q)+p_{g g} b_{g g}(q)$.

We obtain the continuity of the stationary moments $\tau_{*}^{\sigma}$, in uniform/sup norm, in the following (proof in Appendix B):

Theorem 2 Assume $b_{g}, b_{g g}, f_{Q}$ are continuous in $q$. For any $\delta \in \mathcal{D}$ the map $\mathcal{F}$ has an unique fixed point, $\tau_{*}^{\delta}$, if and only if $\rho<1$. Further these fixed points are continuous in supremum norm w.r.t. to parameter $\delta$. From this continuity we get that, $\tau_{*}^{\sigma}=\tau_{*}^{\delta^{\sigma}}$, the stationary moments corresponding to discrete system with $\sigma$ stops, converge to that of the continuous system as $\sigma$ increases to infinity:

$$
\begin{array}{rlrl}
\sup _{q \in \mathcal{C}}\left|\tau_{*}^{\sigma}(q)-\tau_{*}^{\infty}(q)\right| \rightarrow 0 . & \\
\text { Indeed: } \quad & \tau_{*}^{\delta}(|\mathcal{C}|) & =\frac{\delta(|\mathcal{C}|) \alpha^{-1}}{1-\widehat{\rho}(\delta(|\mathcal{C}|) \wedge|\mathcal{C}|)} \text { and } \\
\tau_{*}^{\delta}(q) & =\delta(q) \alpha^{-1}+\tau_{*}^{\delta}(|\mathcal{C}|) \widehat{\rho}(\delta(q) \wedge|\mathcal{C}|) \text { for all } q \\
\text { where } \quad & \widehat{\rho}(q) & :=\lambda \int_{0}^{q} b(y) f_{Q}(y) d y . \diamond
\end{array}
$$

\section{Second Moments:}

Let $C_{n}^{\sigma}(q)$ represent the time taken between the $n^{\text {th }}$ and $n-1^{\text {th }}$ visit of the point $q\left(\delta^{\sigma}(q)\right.$ in case of discrete systems), i.e., the cycle time w.r.t. to the point $q$. Note that

$$
C_{n}^{\sigma}(q)=T_{n}^{\sigma}(q)+\left(T_{n-1}^{\sigma}(|\mathcal{C}|)-T_{n-1}^{\sigma}(q)\right) .
$$

These cycle times are the most important quantities required for further analysis and in this subsection we study its moments. We obtain the convergence of stationary second moments corresponding to $C_{n}^{\sigma}(q)$ as $\sigma \rightarrow \infty$. Let $c_{*}^{2 \sigma}(q)$ represent the (Palm) stationary moments corresponding to $E\left[C_{n}^{\sigma}(q)^{2}\right]$. We obtain their convergence and the exact result is given in the following theorem (proof in Appendix B):

Theorem 3 Assume $b_{g}, b_{g g}, f_{Q}$ are continuous. Then there exists a threshold $\hat{\mathbf{B}}$ on the workload threshold $\hat{B}$, i.e., for service time requirements satisfying $B \leq \hat{\mathbf{B}}$ with probability one, as $\sigma \rightarrow \infty$ the stationary second moments converge uniformly:

$$
\sup _{q \in[0,|C|]}\left|c_{*}^{(2 \sigma)}(q)-c_{*}^{(2 \infty)}(q)\right| \rightarrow 0 . \quad \diamond
$$

\subsubsection{Proof of Theorem 1}

By Theorems 2 and 3, the first stationary moments of $T_{n}^{\sigma}$ and the second order stationary moments of $C_{n}^{\sigma}$, of the discretized polling system, converge towards the corresponding ones of the continuous polling system. The convergence is in supremum norm, i.e., uniformly of the positions $q$ on the circle. We obtain the expression for the expected virtual workload using these moments for both the discrete and continuous

5 In Palm stationary regime, $\tau_{n}^{\sigma}$ are same for all $n$, the common values are represented by $\tau_{*}^{\sigma}$ and hence we get a fixed point operator, $\mathcal{F}$, to represent equation (13). 
polling systems and then using the convergence results of the Theorems 2 and 3 we complete the proof of Theorem 1.

The first two (Palm) stationary moments $E^{0}\left[C_{n}^{\sigma}(q)\right]$ and $E^{0}\left(C_{n}^{\sigma}(q)\right)^{2}$ of $C_{n}^{\sigma}$ defined in $(15)$ are:

$$
\begin{aligned}
c_{*}^{\sigma}(q) & =E^{0}\left[C_{n}^{\sigma}(q)\right]=\tau_{*}^{\sigma}(|\mathcal{C}|), \\
c_{*}^{(2 \sigma)}(q) & =E^{0}\left(C_{n}^{\sigma}(q)\right)^{2} .
\end{aligned}
$$

Convergence of $\tau_{*}^{\sigma}(|\mathcal{C}|)$ is obtained in Theorem 2 while that of $c_{*}^{(2 \sigma)}$ is obtained in Theorem 3. Thus the stationary first moment of the residual of the cycle $C_{n}^{\sigma}(q)$ as seen by a random user in terms of the two Palm moments is given by (see footnote 2),

$$
E\left[C_{R}^{\sigma}(q)\right]=\frac{E^{0}\left(C_{n}^{\sigma}(q)\right)^{2}}{2\left(E^{0}\left[C_{n}^{\sigma}(q)\right]\right)^{2}}=\frac{c_{*}^{(2 \sigma)}(q)}{2\left(\tau_{*}^{\sigma}(|\mathcal{C}|)\right)^{2}} .
$$

Workload due to Globally Gated users: A randomly arriving globally gated user, arriving at point $q$, will have to wait on average for a sum of the following three:

1) the residual of the current cycle (w.r.t. to the globally gated point 0$), E\left[C_{R}^{\sigma}(|\mathcal{C}|)\right]$;

2) $\tau_{*}^{\sigma}(q)$, average time taken by the server to travel from 0 to $\delta^{\sigma}(q)$;

3 ) in a discretized system the user has to wait an extra amount of time to complete service of ${ }^{6}$ : a) the other globally gated users in $\left[\delta^{\sigma}(q), q\right.$ ) (that would be served before him while the server is standing at $\left.\delta^{\sigma}(q)\right)$ and b) the service of all the gated users belonging to the same segment $\left[\delta^{\sigma}(q), \delta^{\sigma}(q)+|\mathcal{C}| / \sigma\right]$, which by Lemma 2 equals:

$$
\begin{gathered}
E\left[\mathcal{T}_{g}\left(\left[\delta^{\sigma}(q), \delta^{\sigma}(q)+\frac{|\mathcal{C}|}{\sigma}\right], C_{n}^{\sigma}\right)\right]+E\left[\mathcal{T}_{g g}\left(\left[\delta^{\sigma}(q), q\right], T_{n-1}^{\sigma}(|\mathcal{C}|)\right)\right] \\
=\tau_{*}^{\sigma}(|\mathcal{C}|)\left(\rho_{g}\left(\delta^{\sigma}(q), \delta^{\sigma}(q)+\frac{|\mathcal{C}|}{\sigma}\right)+\rho_{g g}\left(\delta^{\sigma}(q), q\right)\right) \text { with } \\
\rho_{m}(a, c):=\lambda p_{m} \int_{a}^{c} b_{m}(y) f_{Q}(y) d y \text { for } m=g \text { or } g g .
\end{gathered}
$$

Thus the expected waiting time of such a user for any $\sigma \in \mathbf{N}$ is

$$
\begin{aligned}
E\left[W_{g g}^{\sigma}\right](q)=E[ & \left.C_{R}^{\sigma}(|\mathcal{C}|)\right]+\tau_{*}^{\sigma}(q) \\
& +1_{\{\sigma<\infty\}} \tau_{*}^{\sigma}(|\mathcal{C}|)\left(\rho_{g}\left(\delta^{\sigma}(q), \delta^{\sigma}(q)+\frac{|\mathcal{C}|}{\sigma}\right)+\rho_{g g}\left(\delta^{\sigma}(q), q\right)\right) .
\end{aligned}
$$

Hence, by Little's law ([31]) the density of the stationary number of waiting globally gated users is $\lambda p_{g g} f_{Q}(q) E\left[W_{g g}^{\sigma}\right](q)$ and by Wald's lemma the density of the expected stationary workload corresponding to globally gated users is

$$
\lambda p_{g g} f_{Q}(q) E\left[W_{g g}^{\sigma}\right](q) b_{g g}(q) .
$$

Workload due to Gated users: A randomly arriving gated user, arriving at $q$, has to wait on average for:

1) residual of his own cycle, $E\left[C_{R}^{\sigma}(q)\right]$;

\footnotetext{
6 see arrival position order discussed in subsection 2.4.1 and further note that the gated users are served before the globally gated ones.
} 
2 ) in case of the discretized system till the users placed geographically above him are served which equals (by calculating as done for a globally gated user) $\tau_{*}^{\sigma}(|\mathcal{C}|) \rho_{g}\left(\delta^{\sigma}(q), q\right)$.

Therefore the expected waiting time of a gated user is:

$$
E\left[W_{g}^{\sigma}\right](q)=E\left[C_{R}^{\sigma}(q)\right]+1_{\{\sigma<\infty\}} \tau_{*}^{\sigma}(|\mathcal{C}|) \rho_{g}\left(\delta^{\sigma}(q), q\right) .
$$

Thus by Little's law ([31]) and Wald's lemma the expected stationary workload due to gated users is:

$$
\int_{0}^{|\mathcal{C}|} \lambda p_{g} f_{Q}(q) E\left[W_{g}^{\sigma}\right](q) b_{g}(q) d q .
$$

Total expected stationary virtual workload: Therefore, the total expected stationary virtual workload is given by:

$$
\begin{aligned}
V^{\sigma}= & \lambda \int_{0}^{|\mathcal{C}|}\left(\left(\tau_{*}^{\sigma}(q)+E\left[C_{R}^{\sigma}(|\mathcal{C}|)\right]\right) b_{g g}(q) p_{g g}+E\left[C_{R}^{\sigma}(q)\right] b_{g}(q) p_{g}\right) f_{Q}(q) d q \\
& +1_{\{\sigma<\infty\}} \lambda \tau_{*}^{\sigma}(|\mathcal{C}|) \int_{0}^{|\mathcal{C}|} \nu_{q} f_{Q}(q) d q \text { with } \\
\nu_{q}:= & \rho_{g}\left(\delta^{\sigma}(q), q\right) p_{g} b_{g}(q)+\left(\rho_{g}\left(\delta^{\sigma}(q), \delta^{\sigma}(q)+\frac{|\mathcal{C}|}{\sigma}\right)+\rho_{g g}\left(\delta^{\sigma}(q), q\right)\right) p_{g g} b_{g g}(q) .
\end{aligned}
$$

In the above $V^{\infty}$ represents the expected virtual workload of the random user for the continuous system while $V^{\sigma}$ with $\sigma<\infty$ represents the same for a discrete system. By the Theorems 2 and 3 the terms inside the first integral of (19) (see also the equations (16), (17) and (18)), defining the expected virtual workload, converge towards that of the continuous polling system point-wise and uniformly in $q$. Terms inside the second integral, $\nu_{q}$, converge to zero point-wise and uniformly in $q$ as $\sigma \rightarrow \infty$ :

$$
\sup _{q \in \mathcal{C}} \nu_{q} \leq \lambda\left(p_{g}^{2}\left\|b_{g}\right\|_{\infty}^{2}+p_{g g} p_{g}\left\|b_{g}\right\|_{\infty}\left\|b_{g g}\right\|_{\infty}+p_{g g}^{2}\left\|b_{g g}\right\|_{\infty}^{2}\right)\left\|f_{Q}\right\|_{\infty} \frac{|\mathcal{C}|}{\sigma} .
$$

Thus by bounded convergence theorem, the expected stationary virtual workload of the discretized polling system converges towards that of the continuous polling system as $\sigma \rightarrow \infty$.

Thus the limit of the discrete stationary expected virtual workload, $V_{m i x}^{\sigma}$ of (9), given by (7) represents the the expected stationary virtual workload corresponding to the continuous system. This completes the proof of Theorem 1.

In the coming sections, we analyze an FWLAN using the results of this section.

\section{Ferry Based Wireless Local Area Network (FWLAN)}

Static users are scattered in a geographical area $\Delta$. The network is sparse, there is no direct global connectivity and a message ferry facilitates the basic communication. FWLAN can operate in various architectures (in [30] various architectures are discussed) and we consider here a basic architecture, referred as Sensor Area Network $(\mathrm{SAN})$ in [30]. This configuration ${ }^{7}$ supports only data transfer between the users of

\footnotetext{
7 In a more complicated architecture (referred as Autonomous Network, AUN, in [30]), wherein the ferry also supports the local communication, i.e., the data transfers between users of $\Delta$, is analyzed using continuous polling systems with rerouting in our paper [28].
} 
the area and the outer world via a static gateway, base station (BS). The data transfer from the users of the area to the outer world is called uplink, while that from the outside world to the users of $\Delta$ is called downlink. Every cycle begins at the BS where: 1 ) it dumps to BS the uplink data, collected from the users of LAN in the previous cycle ; 2) collects the downlink data from BS, to transfer in the ongoing cycle to the destined users (when it reaches a point on its path near the intended user).

The ferry "serves" the users: by "serves" a message we mean that the ferry transmits it if the connection is downlink (i.e. the message is destined to a user), or receives it if it is an uplink message.

Ferry's Route : The ferry moves in a closed cyclic path $\mathcal{C}$ repeatedly with constant speed $\alpha$. It stops as and when it encounters an user with a request. Every time it stops and restarts it spends an extra time $t_{a}$ for acceleration. The shuttle continues with its journey after serving the encountered user. Each point in the cyclic path is a potential stop and every stop $q$ in the path is assigned with a set of points $I(q) \subset \Delta$. These sets are assigned based on nearest point basis.

Arrival process: Uplink/Downlink traffic arrives according to an independent marked point processes $\left\{\mathcal{I}_{n}, M_{n}\right\}$, where $\mathcal{T}_{n}$ is the arrival time of the $n$th point and $M_{n}=\left[X_{n}, \eta_{n}\right]$ are the corresponding i.i.d. marks:

$-\mathcal{T}_{n}$ is a Poisson point process with parameter $\lambda$,

- $X_{n}$ is the location of an arrival. It's distribution is given by $P_{X}$.

$-\eta_{n}$ is the size of the message. Its distribution can depend upon $X$ the position of arrival and also on whether it is a uplink or a downlink arrival . It is finite with probability one and its first and second moments (conditioned on the arrival being at point $x$ and further for uplink and downlink arrivals) are given by $\eta_{b}^{u}(x), \eta_{b}^{d}(x), \eta_{b}^{(2 u)}(x), \eta_{b}^{(2 d)}(x)$.

These arrivals ${ }^{8}$ can be uplink, downlink with probabilities $p_{d}, p_{u}=1-p_{d}$ respectively. That is, an uplink request arrives at rate $\lambda^{u}:=p_{u} \lambda$ while the downlink at rate $\lambda^{d}:=$ $p_{d} \lambda$.

We use the following notations. The points in the two dimensional area $\Delta$ are represented by $x$ (if it is a sample point) or $X$ (if it is a random position). The points on the cyclic path $\mathcal{C}$ are represented by $q$ or $Q$. We shall use the superscript $u$ or $d$ to denote uplink or downlink.

Radio channel and service time: The Ferry uses a wireless link to serve the users. It can receive/transmit the messages from/to the users at a distance of $d$ from it at a rate $\kappa(d)$ for some decreasing function $\kappa($.$) . Thus the total time required for$ transmitting a message of size $\eta$, when the user is located at $x \in \Delta$ and is associated with $q(x) \in \mathcal{C}$ is equal to its size divided by the service rate:

$$
B(x)=\frac{\eta}{\kappa(\|q(x)-x\|)} .
$$

The point $x \in \Delta$ is mostly associated with the closest point on the circular path $\mathcal{C}$, i.e., $q(x):=\arg \min _{q \in \mathcal{C}}\|q-x\|$, with $\|$.$\| representing the Euclidean distance.$

8 The analysis of subsection 2.4 requires that at any time there can be at maximum one user waiting at a point. The examples considered in this section satisfy this assumption because of two reasons: i) $P_{X}$ is continuous, ii) The paths considered are sufficiently smooth and hence iii) we will have (will be seen in the coming paragraphs) a $P_{Q}$ that would be continuous. 
The ferry standing at $q \in \mathcal{C}$ has to serve all the users arriving in $I(q)$. Thus the first two moments of the overall time required for uplink service at a point $q \in \mathcal{C}, b_{u}(q)$ and $b_{u}^{(2)}(q)$ are given by ( for all $q \in \mathcal{C}$ ):

$$
\begin{aligned}
b_{u}(q) & :=E[B(X) \mid X \in I(q), \text { uplink }], \\
b_{u}^{(2)}(q) & :=E\left[B(X)^{2} \mid X \in I(q), \text { uplink }\right] .
\end{aligned}
$$

The corresponding for downlink, $b_{d}(q)$ and $b_{d}^{(2)}(q)$, can be defined in a similar way.

Pareto Optimality: All the users are served as the ferry moves on $\mathcal{C}$. Any arrival at a point $x \in I(q) \subset \Delta$ has to wait for time $W(q)$ and the stationary expected values of these waiting times $E[W](q)$ in general can depend upon the point $q \in \mathcal{C}$ standing at which the ferry serves them. We are interested in designing an optimal route (among a given class of trajectories) which minimizes integral of the weighted expected waiting times at all the points in the cyclic path $\mathcal{C}$, as this would give a Pareto optimal solution (see definition of Pareto optimality and other details in Appendix C). Towards this we consider the problem of minimizing

$$
\int_{0}^{|\mathcal{C}|} E[W](q) \zeta(q) d q
$$

where $\zeta(q)$ is a positive weight.

We need to choose appropriate weights $\{\zeta(q)\}$. If $\zeta(q)$ is chosen to be the arrival density to the points that are handled by $q$, i.e. $\lambda P_{I(q)}$, then the integral corresponds to the total expected number of waiting users (by Little's Theorem). If we further multiply the integrand at each point $q$ by the expected service time at that point then we obtain the total expected virtual workload in the system. In this paper the objective function to be minimized will always be the expected virtual workload (which is obtained in section 2 as Theorem 1) and a ferry path which minimized the expected virtual workload is a Pareto optimal path for the multi-objective problem where the expected waiting times at different locations are to be minimized.

Optimizers: We discuss design of optimal ferry paths $\mathcal{C}$ and optimal partitioning of the area into line segments $\left\{I_{q} ; q \in \mathcal{C}\right\}$. The aim of this subsection is to obtain these objects in an optimal way that minimizes the virtual workload. Solving this problem in complete generality will be a very difficult task. Hence we instead obtain optimal ferry path among a special class of ferry paths.

Prior to discussing the optimality issues one first needs to map the FWLAN to a continuous polling system, so that Theorem 1 can be used. This task is taken up immediately.

\subsection{Mapping to a Mixed service Continuous polling system}

We begin with identifying the components of the continuous polling system.

Server and path of the polling system : The ferry represents the server of the polling system. The ferry stops at a point $q$ in its path only when there is a user with a (downlink/uplink) request anywhere on the segment $I(q)$. Thus the entire segment $I(q)$ is modeled as a point on the server's path, in an equivalent continuous polling system.

Service times: The time $t_{a}$ for acceleration is required only when the ferry stops and hence is added to the equivalent service time. An arrival $(\eta, X)$ is associated with 


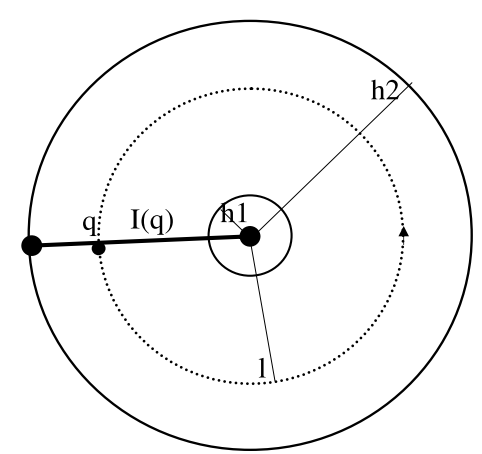

Fig. 1 One Ferry in an annular ring

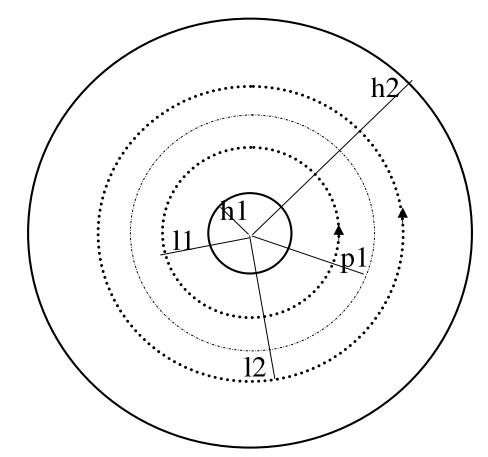

Fig. 2 Two Ferries in an annular ring

the point $q(X):=q$ of the ferry route if $X$ lies in the corresponding segment, i.e., if $X \in I(q)$. Thus service time of an arrival $(\eta, X)$ is given by :

$$
B(X, \eta)=\frac{\eta}{\kappa(|X-q(X)|)}+t_{a}
$$

The expected service times (and the corresponding second moment), for example, for uplink arrivals corresponding to a point $q \in \mathcal{C}$ is

$$
\begin{aligned}
b_{u}(q) & =E[B(X, \eta) \mid q(X)=q]=E_{X}\left[\frac{\eta}{\kappa(|q-X|)} \mid X \in I(q), \text { uplink }\right]+t_{a} . \\
b_{u}^{(2)}(q) & =E\left[B(X, \eta)^{2} \mid q(X)=q\right] \\
& =E_{X}\left[\frac{\eta^{2}}{\kappa(|q-X|)^{2}} \mid X \in I(q), \text { uplink }\right]-t_{a}^{2}+2 t_{a} b_{u}(q) .
\end{aligned}
$$

Service types: Ferry collects all the downlink messages from the BS and distributes the same in the current cycle. Thus the downlink arrivals experience globally gated service, with BS being the global gate point, 0 . On the other hand, the ferry collects uplink data from the users as and when it encounters one on its path. Thus the uplink arrivals experience exhaustive/gated service and therefore $p_{g g}=p_{d}, p_{g}=p_{u}$.

Position of arrival in the 'polling system' : The position of arrival in FWLAN is given by $P_{X}$ which is a distribution over $\Delta$. Every arrival in the segment $I(q)$ marks the arrival at point $q$ of $\mathcal{C}$ in the equivalent polling system. The probability distribution,

$$
P_{Q}(A):=P_{X}\left(\cup_{q \in A} I(q)\right) \text { for any Borel set } A \subset \mathcal{C},
$$

represents this arrival distribution. For example, if $\Delta:=\left\{x \in \mathcal{R}^{2}: h_{1}^{2} \leq|x| \leq h_{2}^{2}\right\}$, $\mathcal{C}=\{q:\|q\|=l\}$ for some $h_{1} \leq l \leq h_{2}$ as in Figure 1, $P_{X} \sim \mathcal{U}(\bar{\Delta})$ (Uniform distribution) and $I(q)=\{x: \angle x=\angle q\}$ (with $\angle x$ representing the angle made by the line joining $0, x$ with the $x$ axis), then $P_{Q}$ will be uniform distribution on $\mathcal{C}$.

Thus the FWLAN can be modeled by a mixed service continuous polling system, Theorem 1 can be applied and the stationary expected virtual workload of the ferry with combined uplink and downlink arrivals can be calculated using (7) for any given cyclic path $\mathcal{C}$ and the corresponding line segments $\left\{I_{q}\right\}_{q \in \mathcal{C}}$. The Theorem can be applied only for those cases which satisfy the hypothesis of the Theorem, like for example 
the service times should be bounded with probability one by $\hat{\mathbf{B}}$ of Theorem 1 . The service times will be bounded as required if: 1) the function $\kappa$ is continuous in $d$ (which usually is the case) and hence its image of a bounded set will be bounded; 2) we will need $P(\eta<\hat{\eta})=1$ with an $\hat{\eta}$ that satisfies, $\hat{\eta} / \min \kappa(d)+t_{a} \leq \hat{\mathbf{B}}$ of Theorem 1 .

In the following subsections, we design optimal FWLAN for a simple example. The ferry moves inside an annular ring $\Delta:=\left\{x \in \mathcal{R}^{2}: h_{1}^{2} \leq|x| \leq h_{2}^{2}\right\}$ of Figures 1 and 2. Ferry moves in a predefined closed cyclic path $\mathcal{C}$ and each point $q$ in the cyclic path is assigned with a line segment $I(q) \subset \Delta$, which is either obtained using the nearest point basis (in subsection 3.2) or is computed in some optimal sense (in subsection 3.3). In subsection 3.3, we consider $N$ circular paths with $N$ ferries each moving in its own circular path. All the paths are optimized jointly so as to further obtain the segments $\{I(q)\}$ optimally.

\subsection{SAN-TAXI example 1: Ferry moving in one circular path}

The ferry moves on one concentric circle of radius $l$ in the annular ring (Figure 1), i.e., $\mathcal{C}_{l}=\left\{q \in \mathcal{R}^{2}:\|q\|=l\right\}$. In this case, the best possible thing is to associate every arrival $(\eta, X)$ with the nearest point of the ferry route, i.e., with the point $Q(X):=$ $\arg \min _{q \in \mathcal{C}_{l}}|q-X|$ of ferry route. Hence the line segments will be angular segments, $I(q)=\{x \in \Delta: L(x)=L(q)\}$ for all $q \in \mathcal{C}_{l}$, (see figure 1 ). We would now like to find the optimal radius of this circle. The variable to be optimized, the radius, takes any value in $\left[h_{1}, h_{2}\right]$ and we are interested in

$$
l^{*}=\arg \min _{l \in\left[h_{1}, h_{2}\right]} V_{S A N-T A X I}^{1}\left(l ; h_{1}, h_{2}\right)
$$

where $V_{S A N-T A X I}^{1}\left(l ; h_{1}, h_{2}\right)$ represents the stationary expected workload in FWLAN when ferry moves in $\mathcal{C}_{l}$, which can be computed using (7). To simplify the computations, in this section, we restrict ourselves to symmetric conditions, i.e.,

- uniform arrivals in annular area, $P_{X} \sim \mathcal{U}(\Delta)$,

- the rate function resulting from the losses in wireless medium considers only the direct path for attenuation (assuming a height difference of 1 unit between the transmitting and the receiving antennae and with path loss factor $\beta$ ):

$$
\kappa(d)=\left(1+d^{2}\right)^{-\beta / 2},
$$

- the distribution of $\eta$ is independent of the location of arrival and the type of arrival (uplink or downlink) with $E[\eta \mid X] \equiv \eta_{b}$ and $E\left[\eta^{2} \mid X\right] \equiv \eta_{b}^{(2)}$ and such that $P(\eta<$ $\hat{\eta})=1$ with an $\hat{\eta} / \kappa\left(d_{\max }\right)+t_{a}<\hat{\mathbf{B}}$, where $d_{\max }:=\left(h_{2}-h_{1}\right) / 2$,

- The uplink and downlink arrivals occur with equal probabilities $p_{g g s}=p_{g s}=1 / 2$.

We note that the hypothesis of the Theorem 1 are satisfied under the symmetric conditions. Since the line segments $\{I(q)\}$ in this case are angular segments, for calculating the moments of the equivalent expected service times of a polling system $\{b(q ; l)\}_{q \in \mathcal{C}_{l}}$ one will require the radius $R=\|X\|$ of the arrival. Under the assumptions of this section, radius $R$ is distributed as $R \sim 2 r d r /\left(h_{2}^{2}-h_{1}^{2}\right)$ and further it is easy to see that 
the the moments of the overall service time will be independent of the position $q \in \mathcal{C}_{l}$ but depend upon the ferry path radius $l$. The service moments are:

$$
\begin{aligned}
b_{l} & =b(q ; l)=\eta_{b} \int_{h_{1}}^{h_{2}}\left(1+(r-l)^{2}\right)^{\beta / 2} \frac{2 r d r}{h_{2}^{2}-h_{1}^{2}}+t_{a}, \\
b_{l}^{(2)} & =b^{(2)}(q ; l)=\eta_{b}^{(2)} \int_{h_{1}}^{h_{2}}\left(1+(r-l)^{2}\right)^{\beta} \frac{2 r d r}{h_{2}^{2}-h_{1}^{2}}-t_{a}^{2}+2 t_{a} b_{l} \quad \text { for all } q \in \mathcal{C}_{l} .
\end{aligned}
$$

For example for $\beta=2$ the above can be computed as,

$$
\begin{aligned}
b_{l} & =\eta_{b}\left(\frac{1}{2}\left(h_{1}^{2}+h_{2}^{2}+2\right)+l^{2}-\frac{4 l\left(h_{1}^{2}+h_{1} h_{2}+h_{2}^{2}\right)}{3\left(h_{1}+h_{2}\right)}\right)+t_{a} \\
b_{l}^{(2)} & =\frac{\eta_{b}^{(2)}}{15\left(h_{2}^{2}-h_{1}^{2}\right)}\left(-5 h_{1}^{6}+24 h_{1}^{5} l-15 h_{1}^{4}\left(3 l^{2}+1\right)+40 h_{1}^{3}\left(l^{3}+l\right)-15 h_{1}^{2}\left(l^{2}+1\right)^{2}\right. \\
& \left.+h_{2}^{2}\left(5 h_{2}^{4}-24 h_{2}^{3} l+15 h_{2}^{2}\left(3 l^{2}+1\right)-40 h_{2}\left(l^{3}+l\right)+15\left(l^{2}+1\right)^{2}\right)\right)-t_{a}^{2}+2 t_{a} b_{l} .
\end{aligned}
$$

Under the simplified assumptions of this section the stationary expected workload for this example can be calculated using $(7)^{9}$ :

$$
V_{S A N-T A X I}^{1}\left(l ; h_{1}, h_{2}\right)=\frac{\lambda b_{l} \lambda b_{l}^{(2)}}{2\left(1-\lambda b_{l}\right)}+\frac{\lambda b_{l}\left|\mathcal{C}_{l}\right| \alpha^{-1}\left(12-\lambda b_{l}\right)}{16\left(1-\lambda b_{l}\right)} .
$$

One has to perform numerical computations using the above formula to obtain the optimal radius and this is done below. However one can get the following asymptotic characteristics of $l^{*}$ from the formula itself: 1 ) as the propagation coefficient $\beta$ tends to zero, both the service moments $b_{l}$ and $b_{l}^{(2)}$ become independent of $l$, stationary expected workload essentially depends upon $l$ only via $\left|\mathcal{C}_{l}\right|$ and hence $l^{*}$ tends to $h_{1}$, i.e., the optimal path for the ferry is the inner circle; 2) as the speed of the ferry, $\alpha$, increases to infinity, the second term in the formula becomes negligible and hence optimal radius will be determined only by the service time moments and so the optimal radius will be above the middle of the ring, i.e., larger than $\left(h_{1}+h_{2}\right) / 2$.

Mixed (Uplink+Downlink) service example : In figure 3 we consider a FWLAN which supports both uplink and downlink services. The parameters of the FWLAN are mentioned in the figure itself and we plot the optimal radius as a function of the outer radius $h_{2}$ of the annular ring. We notice again that the optimal radius tends towards the inner circle either as the speed of the ferry decreases or as $h_{2}$ reduces. We also plot the optimizer of the expected service time,

$$
l_{b}^{*}:=\arg \min _{l \in\left[h_{1}, h_{2}\right]} b_{l},
$$

in the same figure and we note that the optimal radius is close to this optimizer whenever either the annular ring is large or whenever the speed of the ferry is large. This can also be understood by studying the expression for virtual workload (20). Hence in these scenarios one can obtain the optimal radius as (for $\beta=2$ )

$$
l^{*} \approx \frac{2\left(h_{1}^{2}+h_{2}^{2}+h_{1} h_{2}\right)}{3\left(h_{1}+h_{2}\right)} .
$$

\footnotetext{
9 The equation below is different from the one in [29], because of the correction in (7).
} 


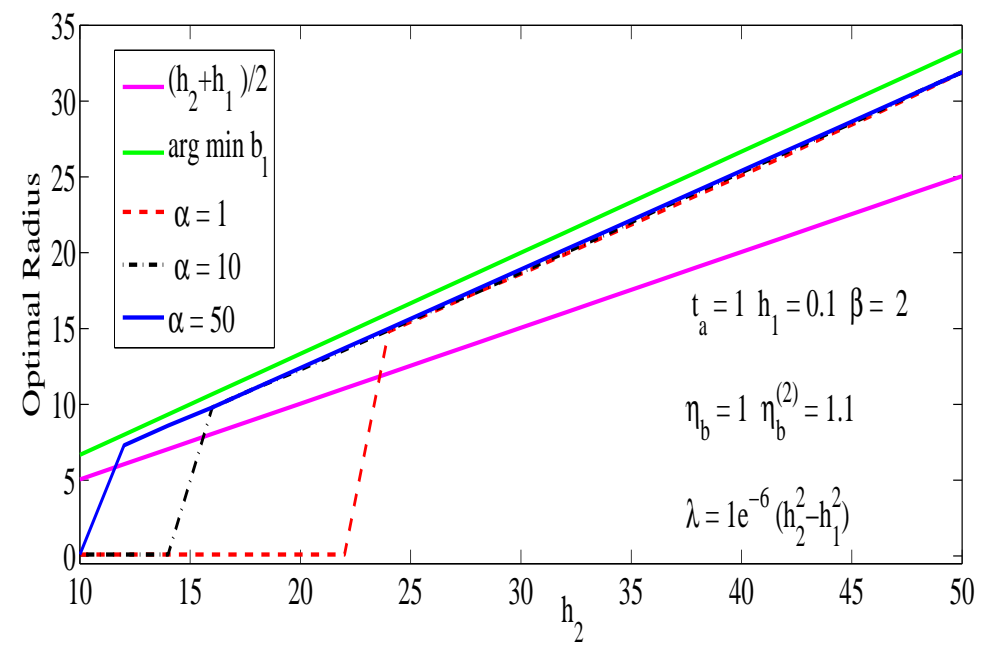

Fig. 3 Optimal orbit radius for a ferry moving continuously in a annular ring (Uplink and Downlink)

\subsection{SAN-TAXI example 2: $N$ servers moving simultaneously in the annular area}

When the annular ring spans over a large area, a ferry moving in one circular path may not be optimal. Hence we would like to consider a ferry moving in $N$ circular paths before completing the area. To make things simpler we assume that there will be $N$ ferries moving simultaneously (but independent of each other) with the same speed $\alpha$ respectively on circular paths of radii $l_{1}, l_{2}, \cdots, l_{N}$ (see figure 2 ). Without loss of generality we also assume that the BS is placed on the entire segment $\{x: \angle x=0\}$, i.e., any ferry communicates with the BS instantaneously the moment it touches the angle 0 . Throughout this paper we are neglecting the time taken for communication (e.g., the communication happens at high speed using a wired link) between the BS and the ferry(ies). Let $\mathcal{C}_{n}:=\left\{q:\|q\|=l_{n}\right\}$ and let $\mathcal{B}(x, p):=\{y:\|y-x\|<p\}$ represent the open ball, while $\overline{\mathcal{B}}(x, p)$ represent its closure. The first ferry caters to the users in the annular ring $\overline{\mathcal{B}}\left(0, p_{1}\right)-\mathcal{B}\left(0, h_{1}\right)$ while the last one covers the annular ring $\overline{\mathcal{B}}\left(0, h_{2}\right)-\mathcal{B}\left(0, p_{N-1}\right)$. Any intermediate $n^{\text {th }}$ ferry covers the area $\overline{\mathcal{B}}\left(0, p_{n}\right)-$ $\mathcal{B}\left(0, p_{n-1}\right)$. Let $p_{0}:=h_{1}$ and $p_{N}:=h_{2}$. In this subsection we choose optimally $\mathbf{p}:=$ $\left[p_{1}, p_{2}, \cdots, p_{N-1}\right]^{T}$ and $\mathbf{l}:=\left[l_{1}, l_{2}, \cdots, l_{N}\right]^{T}$, which in turn optimally defines the line segments $\left\{I_{n}(q) ; q \in \mathcal{C}_{n}\right.$, for all $\left.n\right\}$ as

$$
I_{n}(q)=\left\{x \in \overline{\mathcal{B}}\left(0, p_{n}\right)-\mathcal{B}\left(0, p_{n-1}\right): \angle q=\angle x\right\}
$$


We map each ferry by a separate continuous polling system. With these, the moments of the overall service times for the $n^{\text {th }}$ ferry is

$$
\begin{aligned}
& b_{n, \mathbf{p}, \mathbf{l}}=\eta_{b} \int_{p_{n-1}}^{p_{n}}\left(1+(r-l)^{2}\right)^{\beta / 2} \frac{2 r d r}{p_{n}^{2}-p_{n-1}^{2}}+t_{a} \text { and } \\
& b_{n, \mathbf{p}, \mathbf{l}}^{(2)}=\eta_{b}^{(2)} \int_{p_{n-1}}^{p_{n}}\left(1+(r-l)^{2}\right)^{\beta} \frac{2 r d r}{p_{n}^{2}-p_{n-1}^{2}}-t_{a}^{2}+2 t_{a} b_{n, \mathbf{p}, \mathbf{1}}
\end{aligned}
$$

Using these moments one can estimate $\left\{V_{S A N-T A X I}^{1}\left(l_{n} ; p_{n-1}, p_{n}\right)\right\}$, the $N$ virtual workloads as a function of the vectors $\mathbf{p}, \mathbf{l}$. We now propose to choose the optimal ferry paths (and line segments $\{I(q)\})$ by :

$$
\left(\mathbf{p}^{*}, \mathbf{l}^{*}\right)=\arg \min _{\mathbf{p}, \mathbf{l}} \sum_{n=1}^{N} \frac{p_{n}^{2}-p_{n-1}^{2}}{h_{2}^{2}-h_{1}^{2}} V_{S A N-T A X I}^{1}\left(l_{n} ; p_{n-1}, p_{n}\right) .
$$

In the following we consider an example with 2 ferries moving in the annular area and supporting mixed service. The results are in Table 1 . We obtain the optimal $\mathbf{l}^{*}, p^{*}$ for different values of the outer radius $h_{2}$ and the common speed of the ferries $\alpha$ while the rest of the parameters are kept constant. We see again that the optimal radii of the ferry routes move towards the inner circle as the speed of the vehicle reduces and this effect is pronounced as the the radius of the outer circle, $h_{2}$, decreases. This effect is also seen in the case of single server and can be partially explained using the expression $V_{S A N-T A X I}(20)$ for the virtual workload:

- $V_{S A N-T A X I}$ depends upon the radius $l$ via the moments of the effective service times $b^{(1)}, b^{(2)}$ and the circumference $|\mathcal{C}|$. Virtual workload $V_{S A N-T A X I}(20)$ is directly proportional to all three factors.

- The influence of $l$ on the two moments depends upon the path loss factor $\beta$. If $\beta$ is close to zero the moments are almost independent of $l$ while the optimal $l$ (optimal for the service moments) will be above the center of the annular ring for practical path loss factors. For practical values of path loss factors, the two moments reduce as $l$ increases from the inner radius.

- However the circumference $|\mathcal{C}|$ has a contrast effect, it increases linearly with $l$.

- For large values of speed $\alpha$, the influence of $|\mathcal{C}|$ on $V_{S A N-T A X I}$ reduces and hence the optimal radii will be above the center of the annular ring.

- For smaller values of speed $\alpha$, we see the reverse effect.

- The area of the annular ring also influences, with larger areas the moments (especially the second) will be large and hence the influence of the moments on $V_{S A N-T A X I}$ will be larger than that of the circumference $|\mathcal{C}|$. And so, we see from the bottom rows of the table that, the optimal radii are away from the inner circle even for lower speeds.

\section{FWLAN with two base stations}

We now consider a LAN equipped with two base stations and a moving ferry. These base stations have also to be placed in optimal locations along with the design of optimal ferry routes. In this section, we consider downlink service alone. Before we proceed with FWLAN examples with two base stations, we note here that many of the 


\begin{tabular}{|c|c|c|c|}
\hline & $\begin{array}{c}\alpha=100 \\
h_{2}\end{array}$ & $\begin{array}{c}\alpha=10 \\
\left(l_{1}^{*}, p^{*}, l_{2}^{*}\right)\end{array}$ & $\begin{array}{c}\alpha=1 \\
\left(l_{1}^{*}, p^{*}, l_{2}^{*}\right)\end{array}$ \\
\hline 10 & $6.3,7.7,8.8$ & $6.1,7.7,8.6$ & $6,7.8,8.6$ \\
\hline 15 & $7.9,10.4,12.7$ & $7.8,10.6,12.7$ & $7.8,10.8,12.8$ \\
\hline 20 & $9.5,13.1,16.7$ & $9.6,13.5,16.8$ & $9.6,13.8,16.9$ \\
\hline 25 & $11.1,15.8,20.6$ & $11.3,16.3,20.8$ & $11.4,16.7,20.9$ \\
\hline 30 & $12.8,18.5,24.6$ & $13,19,24.7$ & $13.2,19.5,24.9$ \\
\hline 35 & $14.4,21.1,28.5$ & $14.6,21.5,28.6$ & $14.9,22.1,28.8$ \\
\hline
\end{tabular}

Table 1 Example : 2 Ferries moving in annular area with $\beta=2 h_{1}=5, t_{a}=10, \eta_{b}=2$, $\eta_{b}^{(2)}=4.4, \lambda=1 e^{-2}$

two base station examples can result in a special case of service called elevator polling (see [19] which describes the scheme for discrete polling system). This polling system will have 'fair' behavior : the expected waiting times at all the points in the server route will be equal irrespective of whether the arrivals are symmetric or non symmetric. This 'fair' behavior is a welcome feature and it might be advantageous to consider the paths and the BS locations that can result in this behavior. In the subsection 2.3 we derived the expression for the stationary expected waiting time in a continuous polling system with elevator gating. These results are applied to some interesting FWLAN examples with two base stations in the following.

\subsection{Ferry moving in a straight line in Rectangular area}

We consider a rectangular area $\Delta=[-l, l] \times[-d, d]$. A ferry moves in the horizontal straight line as shown in Figure 4. There are two base stations, each one of them is located at the end of the ferry path. The ferry, say, starts its cycle by collecting all the downlink data from the BS on the left, tags the users for whom the downlink data has to be distributed, moves towards right with speed $\alpha$, distributes the data to all the tagged users. Once reaching the BS on the right it collects all the data from the BS and then moves left. This procedure repeats. We analyze this FWLAN using the elevator polling system of subsection 2.3.

Let the base stations be placed respectively at $\left(p_{1}, 0\right),\left(p_{2}, 0\right)$. Let $\mathbf{p}:=\left(p_{1}, p_{2}\right)$. In this case the ferry path is given by $\mathcal{C}_{\mathbf{p}}=\left\{(q, 0): p_{1} \leq q \leq p_{2}\right\}$. The partitions are calculated based on the nearest distance criterion.

$$
I(q)=I(q, 0)=\left\{\begin{aligned}
&\{q\} \times[-d, d] p_{1}<q<p_{2} \\
& {\left[-l, p_{1}\right] \times[-d, d] q=p_{1} } \\
& {\left[p_{2}, l\right] \times[-d, d] q=p_{2} }
\end{aligned}\right.
$$

Note in this case that, both the ends of the ferry path cover not just a line segment but a rectangular segment. We make simplifying assumptions as in section 3. Under these assumptions

$$
\begin{aligned}
& b(q ; \mathbf{p})=\left\{\begin{array}{lr}
\eta_{b} \int_{-d}^{d}\left(1+y^{2}\right)^{\beta / 2} \frac{d y}{2 d}+t_{a} & p_{1}<q<p_{2} \\
\eta_{b} \int_{-l}^{p_{1}} \int_{-d}^{d}\left(1+y^{2}+\left(p_{1}-x\right)^{2}\right)^{\beta / 2} \frac{d x d y}{4 l d}+t_{a} & q=p_{1} \\
\eta_{b} \int_{p_{2}}^{l} \int_{-d}^{d}\left(1+y^{2}+\left(p_{2}-x\right)^{2}\right)^{\beta / 2} \frac{d x d y}{4 l d}+t_{a} & q=p_{2}
\end{array}\right. \\
& b^{(2)}(q ; \mathbf{p})-2 b(q ; \mathbf{p}) t_{a}+t_{a}^{2}=\left\{\begin{array}{lr}
\eta_{b}^{(2)} \int_{-d}^{d}\left(1+y^{2}\right)^{\beta} \frac{d y}{2 d} & p_{1}<q<p_{2} \\
\eta_{b}^{(2)} \int_{-l}^{p_{1}} \int_{-d}^{d}\left(1+y^{2}+\left(p_{1}-x\right)^{2}\right)^{\beta} \frac{d x d y}{4 l d} q=p_{1} \\
\eta_{b}^{(2)} \int_{p_{2}}^{l} \int_{-d}^{d}\left(1+y^{2}+\left(p_{2}-x\right)^{2}\right)^{\beta} \frac{d x d y}{4 l d} q=p_{2} .
\end{array}\right.
\end{aligned}
$$


Note here that even under symmetric assumptions the overall service moments are not equal at all points, thus resulting in an asymmetrical polling system. With the above the overall service moments are given by,

$$
\begin{aligned}
b_{\mathbf{p}} & =2 d\left(l-p_{2}\right) b\left(p_{1} ; \mathbf{p}\right)+2 d\left(p_{1}+l\right) b\left(p_{2} ; \mathbf{p}\right)+2 d\left(p_{2}-p_{1}\right) b\left(p_{1}+; \mathbf{p}\right) \\
b_{\mathbf{p}}^{(2)} & =2 d\left(l-p_{2}\right) b^{(2)}\left(p_{1}\right)+2 d\left(p_{1}+l\right) b^{(2)}\left(p_{2}\right)+2 d\left(p_{2}-p_{1}\right) b^{(2)}\left(p_{1}+; \mathbf{p}\right) .
\end{aligned}
$$

In the above by $b\left(p_{1}+; \mathbf{p}\right)$ we meant the expected value at any point with its $\mathrm{x}$ component greater than $p_{1}$. As the expected weighting times in this case are equal at all the points, it will be Pareto optimal to optimize the expected weighting times themselves. Further in this symmetric case it is easy to see that $p_{1}^{*}=-p_{2}^{*}$. Thus,

$$
p_{2}^{*}=\arg \min _{0<p_{2} \leq d} \frac{\lambda b_{-p_{2}, p_{2}}^{(2)}}{2\left(1-\lambda b_{-p_{2}, p_{2}}\right)}+2 p_{2} \alpha^{-1} \text {. }
$$

Note here that optimal choice of $p_{2}^{*}$ optimally places the two base stations as well as optimally designs the ferry path. In this example, it is easy to see that : (1) As the speed of the ferry $\alpha$ increases, the $p_{2}^{*}$ moves towards $l$, i.e., if the ferry can move at large speeds, it is optimal to place the base stations at both the edges of the area and allow the ferry to traverse the entire width of $\Delta$. This conclusion is also true whenever the path loss factor $\beta$ is large. (2) If the ferry moves at moderate speeds and or the path loss factor is small $p_{2}^{*}$ reduces. Thus for small path loss factors, the ferry can move in shorter line segments and cover the entire area optimally.

We simplified the problem substantially by considering that the ferry moves in a horizontal line placed at the center of the area. This simplification is good as long as the arrivals are uniform and the file sizes are distributed the same way at all points. For asymmetrical arrivals this choice of ferry route may not be good. In this case it might be better to consider any general straight line passing through points $\left(p_{1}, p_{2}\right)$ and $\left(p_{3}, p_{4}\right)$ as ferry path. The base stations are placed once again at both ends of the ferry path. One can estimate the expected waiting time in terms of the parameter $\mathbf{p}:=\left(p_{1}, p_{2}, p_{3}, p_{4}\right)$ in a similar way and choose $\mathbf{p}^{*}$ optimally. This $\mathbf{p}^{*}$ places the base stations optimally and designs the ferry routes optimally in more generality. If the breadth of $\Delta, 2 d$, is large it might be better to consider zig zag ferry paths (Figure 5 ) with two base stations placed at $\left(p_{1}, p_{2}\right)$ and $\left(p_{3}, p_{4}\right)$.

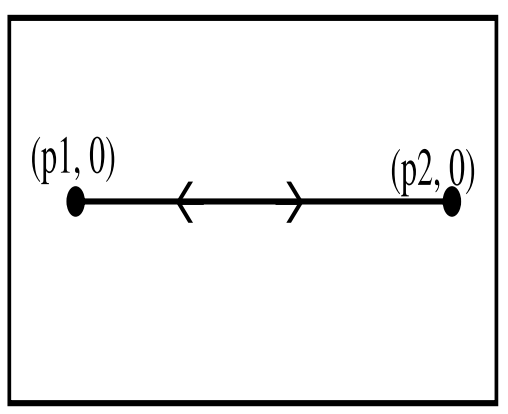

Fig. 4 One Ferry in Rectangular area with two base stations

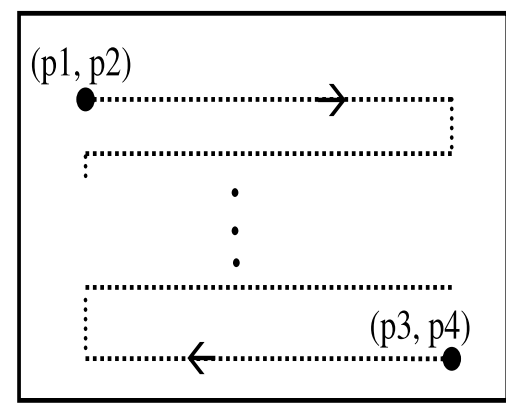

Fig. 5 More general paths for the ferry with two base stations 
Acknowledgments

The reviewers have pointed out a gap in the proof of Theorem 3 (of the revision 1) and we corrected the same in the final manuscript. We are thankful to the reviewers for pointing out the gap and allowing us to correct the same.

\section{Conclusions}

We study continuous polling systems that cater to non-standard variants of gating disciplines like, globally gated service, gated service, mixed service (a mixture of globally gated and gated service disciplines) and elevator service disciplines. We obtain an expression for the expected stationary workload. We obtain this result under more general conditions than the usual symmetric conditions. We come up with a way of discretization such that the available Pseudo conservation laws of discrete polling systems can be utilized for obtaining the results for the continuous counterparts. We expressed the expected workload as a parametrized function of moment fixed points. The later are some stationary moments obtained as fixed points of a function defined on spaces of left continuous functions with right limits equipped with supremum norm and which are further parametrized by the number of discretization levels. We show the required convergence via the continuous dependence of the fixed points on the parameter. This way we obtained a common expression, which represents the expected virtual workload for continuous as well as the discretized polling systems, at different values of the parameter. We then showed the continuity of the expected virtual workload with respect to the parameter and hence obtained the expected virtual workload for the continuous system as the limit of the expected virtual workloads of the discrete systems, when the levels of discretization tend to infinity. We applied these results to a wireless LAN in which a ferry assists data transfer between the users of the network and a base station which acts as a gateway to the external world. We provided the analytical expressions for the expected virtual workload in a system of FWLAN, taking into account the radio channel considerations, which allowed us to optimize the trajectories of the Ferry. We covered both uplink, downlink as well as their combination. Minimizing the virtual workload results in a Pareto minimization of the expected waiting times in the system. We have further presented some extensions to the case of several base stations and to routing of several ferries. We observe that by using two base stations, one can achieve fairness : the expected waiting times are independent of the positions of arrivals.

In [28], using similar approach we analyzed an FWLAN, in which the ferry also supports local communication. This FWLAN is analyzed using a continuous polling system with rerouting, whose performance is once again obtained using the discretization approach. One can further explore this discretization approach.

Appendix A: Limit of the Virtual Workload, $V_{m i x}^{\sigma}$

Proof of Lemma 1: Some terms of (9) are independent of $\sigma$ :

$$
\rho=\sum_{i=1}^{2 \sigma} \lambda_{i} b_{i}=\lambda \int_{0}^{|\mathcal{C}|} b(q) P_{Q}(d q)=\lambda \bar{b}
$$




$$
\begin{aligned}
\sum_{i=1}^{2 \sigma} \lambda_{i} b_{i}^{(2)} & =\lambda \bar{b}^{(2)} \text { and } \\
\sum_{i=1}^{\sigma} \rho_{2 i} & =\lambda p_{g g} E[B \mid \text { globally gated }]=\lambda p_{g g} \bar{b}_{g g} .
\end{aligned}
$$

Recall $i^{\sigma}=(i-1)|\mathcal{C}| / \sigma$, note $\delta^{\sigma}(q)=i^{\sigma}$ when $q \in I_{i}$ and with these,

$$
\begin{aligned}
\sum_{i}^{\sigma} \rho_{2 i} \frac{(i-1)|\mathcal{C}| \alpha^{-1}}{\sigma}=\lambda p_{g g} \alpha^{-1} \sum_{i}^{\sigma} E\left[b_{g g}(Q) 1_{\left\{Q \in I_{i}\right\}}\right] i^{\sigma} \\
=\lambda p_{g g} \alpha^{-1} \sum_{i}^{\sigma} b_{g g}\left(i^{\sigma}\right) f_{Q}\left(i^{\sigma}\right) \frac{|\mathcal{C}|}{\sigma} i^{\sigma} \\
+\lambda p_{g g} \alpha^{-1} \int_{0}^{|\mathcal{C}|}\left(b_{g g}(q) f_{Q}(q)-b_{g g}\left(\delta^{\sigma}(q)\right) f_{Q}\left(\delta^{\sigma}(q)\right)\right) \delta^{\sigma}(q) d q \\
\stackrel{\sigma \rightarrow \infty}{\rightarrow} \lambda p_{g g} \alpha^{-1} \int_{0}^{\sigma} q b_{g g}(q) f_{Q}(q) d q=\lambda p_{g g} \alpha^{-1} E\left[Q b_{g g}(Q)\right]
\end{aligned}
$$

The term (22) is a Riemann sum and hence converges to the integral (24) while the second term (23) converges to zero by continuity of the functions $b_{g g}, f_{Q}$ via Bounded Convergence Theorem (BCT). Similarly, excluding the terms which converge to zero by BCT as in (23),

$$
\begin{aligned}
\sum_{i=1}^{2 \sigma} \rho_{i}^{2} & \approx \frac{\lambda|\mathcal{C}|}{\sigma} \sum_{i=1}^{\sigma}\left(p_{g} b_{g}^{2}\left(i^{\sigma}\right)+p_{g g} b_{g g}^{2}\left(i^{\sigma}\right)\right) f_{Q}^{2}\left(i^{\sigma}\right) \frac{|\mathcal{C}|}{\sigma} \\
& \rightarrow 0 \int_{0}^{\sigma}\left(p_{g g} b_{g g}^{2}(q)+p_{g} b_{g}^{2}(q)\right) f_{Q}^{2}(q) d q=0 .
\end{aligned}
$$

Following similar logic, with $\left.\widehat{b}_{g}(q):=E\left[b_{g}(Q) 1_{\{Q \leq q\}}\right]\right)$ and from $(21)$ :

$$
\begin{aligned}
\sum_{i}^{\sigma} \rho_{2 i} \sum_{j \leq 2 i} \rho_{j} & =\sum_{i=1}^{\sigma} \rho_{2 i} \sum_{j \leq i} \rho_{2 j}+\sum_{i=1}^{\sigma} \rho_{2 i} \sum_{j \leq i} \rho_{2 j-1} \\
& =\frac{1}{2}\left(\sum_{i=1}^{\sigma} \rho_{2 i}\right)^{2}+\frac{1}{2} \sum_{i}^{\sigma} \rho_{2 i}^{2}+\lambda^{2} p_{g g} p_{g} \sum_{i=1}^{\sigma} b_{2 i} l_{i} \sum_{j \leq i} b_{2 j-1} l_{j} \\
& \rightarrow \frac{\lambda^{2}}{2}\left(\left(p_{g g} \bar{b}_{g g}\right)^{2}+p_{g g} p_{g} E\left[b_{g g}(Q) \widehat{b}_{g}(Q)\right]\right)
\end{aligned}
$$

By independence $E\left[b_{g g}(Q) \widehat{b}_{g}(Q)\right]=\bar{b}_{g g} \widehat{\widehat{b}}_{g}$ and hence from limits (21)-(26), the limit of $V_{m i x}^{\sigma}(9)$ matches with the formula (7) of the Theorem 1.

\section{Appendix B}

Proof of Lemma 2: Let $T(q) \equiv T$, with $E[T]=\tau$. In this case, $\mathcal{T}([a, c], T)$ is sum of the service times of all the arrivals in the interval $[0, T]$ that occurred in the segment 
$[a, c] \subset[0,|\mathcal{C}|]$. Let $\mathcal{N}$ represent the number of those Poisson arrivals that belong to segment $[a, c]$ and that arrived during the $[0, T]$ interval. In this case,

$$
E[\mathcal{N}]=E[E[\mathcal{N} \mid T]]=\lambda P_{Q}([a, c]) E[T]=\lambda \tau \int_{a}^{c} f_{Q}(q) d q
$$

Let $\left\{B_{i}\right\}_{i<\mathcal{N}}$ represent the service times of these $\mathcal{N}$ arrivals. Any arrival can fall in point $q$ according to distribution $P_{Q}(d q)=f_{Q}(q) d q$ and the conditional expected service time of the arrival conditioned on the point of arrival is $b(q)$ and so,

$$
E\left[B_{i} \mid \text { Arrival in }[a, c]\right]=\frac{1}{P_{Q}([a, c])} \int_{a}^{c} b(q) f_{Q}(q) d q
$$

Thus by Wald's lemma ${ }^{10}$,

$$
E[\mathcal{T}([a, c], T)]=E\left[\sum_{i=1}^{\mathcal{N}} B_{i}\right]=E[\mathcal{N}] E\left[B_{i}\right]=\lambda \tau \int_{a}^{c} b(q) f_{Q}(q) d q
$$

Now we consider the other case, i.e., let every sample path of the the random function $T$ be a monotone increasing function of $q \in[a, c]$. The proof for $T$, monotone decreasing, follows in similar lines. For every $M$, define step function,

$$
\delta^{M}(q)=a+\frac{i}{M} \text { if } q \in a+\left[\frac{i}{M}, \frac{i+1}{M}\right) .
$$

Note that by monotonicity of $T$,

$$
\begin{aligned}
& \underline{T}^{M}(q):=T\left(\delta^{M}(q)\right) \leq T(q) \text { and } \\
& \bar{T}^{M}(q):=T\left(\delta^{M}(q)+\frac{1}{M}\right) \geq T(q) .
\end{aligned}
$$

In the following the arrivals and their service times are independent ${ }^{11}$ of $T, \bar{T}^{M}$ and $\underline{T}^{M}$. More arrivals can occur during more time and hence workload during larger time will be larger. Hence and further using (27)

$$
\begin{aligned}
& E[\mathcal{T}([a, c], T)] \leq E\left[\mathcal{T}\left([a, c], \bar{T}^{M}\right)\right] \\
& \quad=\lambda \sum_{i=0}^{M-1} \tau\left(a+\frac{i}{M}\right) \int_{a+i / M}^{a+(i+1) / M} f_{Q}(q) b(q) d q
\end{aligned}
$$

\footnotetext{
10 For applying Wald's lemma we need certain independence assumptions. These assumptions are satisfied in this proof (whenever Wald's lemma is applied) because of the following: the service times (and the number) of incoming arrivals are independent of the system evolution till the time stamp at which they are served. For example, the number of arrivals (or those tagged in case of globally gated service) at around a point say $q$ (i.e., the users awaiting service in segment $[q, q+d q]$ ) does not influence the system evolution till the server reaches point $\delta^{\sigma}(q)$, standing at which it would serve the awaiting users, i.e., till time $T_{n}^{\sigma}(q)$. To put it the other way, for example, $T_{n}^{\sigma}(q)$ is a stopping time for that sampled arrival process (sampled from the original gated Poisson arrival process), in which the users land in segment $[q, q+d q]$ and which originated during the time interval $C_{n}^{\sigma}(q)$.

11 The independence assumption holds for lower bound $\underline{T}^{M}$ and the actual time $T$ readily because of the reasons given in footnote 10. An upper bound is obtained by calculating the independent arrivals that would have arrived in a bigger interval i.e., $\bar{T}^{M}$.
} 


$$
\begin{aligned}
& \leq \lambda \sum_{i=0}^{M-1} f_{Q}\left(a+\frac{i}{M}\right) b\left(a+\frac{i}{M}\right) \tau\left(a+\frac{i}{M}\right) \frac{1}{M} \\
& \quad+\lambda \int_{a}^{c} \tau\left(\delta^{M}(q)\right)\left(f_{Q}(q) b(q)-b\left(\delta^{M}(q)\right) f_{Q}\left(\delta^{M}(q)\right)\right) d q \\
& \quad \rightarrow \lambda \int_{a}^{c} f_{Q}(q) \tau(q) b(q) d q \text { as } M \rightarrow \infty .
\end{aligned}
$$

The last limit is obtained because the partial sums define Riemann sums and they converge to the integral and because the second integral goes to zero by uniform continuity of $b, f_{Q}$ functions (continuous function on compact intervals are uniformly continuous). Similarly using the lower bound on the time $T, \underline{T}^{M}$, we get,

$$
E[\mathcal{T}([a, c], T)] \geq \lim _{M \rightarrow \infty} E\left[\mathcal{T}\left([a, c], \underline{T}^{M}\right)\right]=\lambda \int_{a}^{c} f_{Q}(q) \tau(q) b(q) d q .
$$

Hence,

$$
E[\mathcal{T}([a, c], T)]=\lambda \int_{a}^{c} f_{Q}(q) \tau(q) b(q) d q . \quad \diamond
$$

Proof of Theorem 2: The operator $\mathcal{F}$ given by (14) is reproduced here.

$$
\mathcal{F}(\tau ; \delta)(q):=\delta(q) \alpha^{-1}+\lambda \tau(|\mathcal{C}|) \int_{0}^{\delta(q) \wedge|\mathcal{C}|} b(y) f_{Q}(y) d y
$$

We are interested in it's fixed point. The function is clearly jointly continuous in $\tau, \delta$. Further,

$$
\mathcal{F}\left(\tau_{1} ; \delta\right)(q)-\mathcal{F}\left(\tau_{2} ; \delta\right)(q)=\lambda\left(\tau_{1}(|\mathcal{C}|)-\tau_{2}(|\mathcal{C}|)\right) \int_{0}^{\delta(q) \wedge|\mathcal{C}|} b(y) f_{Q}(y) d y
$$

Thus for all $\delta$

$$
\left\|\mathcal{F}\left(\tau_{1} ; \delta\right)-\mathcal{F}\left(\tau_{2} ; \delta\right)\right\|_{\infty} \leq \rho\left\|\tau_{1}-\tau_{2}\right\|_{\infty}
$$

Thus whenever $\rho<1$, we have a fixed point of the operator $\mathcal{F}$ for all $\delta$ and hence the existence of unique stationary first moments. Under this condition, we further see by parametrized contraction mapping theorem ([32]) that the unique fixed point $\tau_{*}^{\delta}$ is continuous in $\delta$.

In fact from equation (14), when evaluated at $q=|\mathcal{C}|$, we find that:

$$
\tau_{*}^{\delta}(|\mathcal{C}|)=\frac{\delta(q) \alpha^{-1}}{1-\lambda \int_{0}^{\delta(|\mathcal{C}|) \wedge|\mathcal{C}|} b(y) f_{Q}(y) d y} .
$$

Note that

$$
\sup _{\delta \in \mathcal{D}} \lambda \int_{0}^{\delta(|\mathcal{C}|) \wedge|\mathcal{C}|} b(y) f_{Q}(y) d y=\lambda \int_{0}^{|\mathcal{C}|} b(y) f_{Q}(y) d y=\rho
$$

and thus the stationary moments exist if and only if $\rho<1$. Under this condition, one can solve the fixed point equation and complete the proof of Theorem 2 . 
Proof of Theorem 3: The cycle time $C_{n}^{\sigma}$ defined in (15) can be rewritten in the following fashion and via this expression we obtain the convergence of the required second moments (note $C_{n}^{\sigma}(0)=C_{n-1}^{\sigma}(|\mathcal{C}|)$ almost surely by continuity arguments):

$$
\begin{aligned}
C_{n}^{\sigma}(q) & =|\mathcal{C}| \alpha^{-1}+U_{n}^{\sigma}(q)+\mathcal{T}_{g}\left(\left[0, \delta^{\sigma}(q)\right), C_{n}^{\sigma}\right)+\mathcal{T}_{g g}\left(\left[0, \delta^{\sigma}(q)\right), C_{n}^{\sigma}(0)\right) \\
U_{n}^{\sigma}(q) & \left.=\mathcal{T}_{g g}\left(\left[\delta^{\sigma}(q),|\mathcal{C}|\right), C_{n-1}^{\sigma}(0)\right)+\mathcal{T}_{g}\left(\left[\delta^{\sigma}(q),|\mathcal{C}|\right), C_{n-1}^{\sigma}\right)\right) .
\end{aligned}
$$

Required Spaces: We first identify appropriate complete metric spaces in which the above quantities reside and whose norm gives the required convergence. We start with some definitions/notations.

Let $(\Omega, \mathcal{F}, P)$ be any probability space.

Let $\|.\|_{2}$ be the $L^{2}$ norm, i.e., $\|\psi\|_{2}:=E\left[\psi^{2}\right]$ for any random variable $\psi$.

Let $\mathcal{D}$ represent the space of left continuous functions on $[0,|\mathcal{C}|]$ with right limits and equipped with supremum norm, $\|f\|_{\infty}:=\sup _{q \in[0,|\mathcal{C}|]}|f(q)|$.

Let

$$
\begin{aligned}
\Psi & :=\{\text { measurable functions } \psi:[0,|\mathcal{C}|] \times \Omega \rightarrow \mathcal{R} \text { such that } \\
\text { the map } \left.q \mapsto\|\psi(q, .)\|_{2} \in \mathcal{D}\right\} \text { where } & \mapsto \psi(q, w)^{2} d P(\omega)=E\left[\psi(q, .)^{2}\right] .
\end{aligned}
$$

Define the following norm on this space:

$$
\|\psi\|_{\infty, 2}:=\sup _{q \in[0,|\mathcal{C}|]}\|\psi(q, .)\|_{2} \text { for any } \psi \in \Psi .
$$

By Lemma $3\left(\Psi,\|\cdot\|_{\infty, 2}\right)$ is a Banach space.

Contraction Mappings and fixed points: It is easy to see that $\left(C_{n}^{\sigma}, U_{n}^{\sigma}\right)$ defined in (29)-(30) reside ${ }^{12}$ in $\Psi$ for all $\sigma$. It is also easy to see that these under stationarity form the fixed point of the following function, defined point-wise (i.e., for every $q$ ) by:

$$
\begin{aligned}
& \Theta_{C}^{\sigma}\left(\psi_{c}, \psi_{u} ; \sigma\right)(q)=|\mathcal{C}| \alpha^{-1}+\sqrt{\hat{B}} \psi_{u}(q)+\mathcal{T}_{g}\left(\left[0, \delta^{\sigma}(q)\right], \psi_{c}\right)+\mathcal{T}_{g g}\left(\left[0, \delta^{\sigma}(q)\right], \psi_{c}(|\mathcal{C}|)\right) \\
& \Theta_{U}^{\sigma}\left(\psi_{c}, \psi_{u} ; \sigma\right)(q)=\frac{1}{\sqrt{\hat{B}}} \mathcal{T}_{g g}\left(\left[\delta^{\sigma}(q),|\mathcal{C}|\right], \psi_{c}(|\mathcal{C}|)\right)+\frac{1}{\sqrt{\hat{B}}} \mathcal{T}_{g}\left(\left[\delta^{\sigma}(q),|\mathcal{C}|\right], \psi_{c}\right) .
\end{aligned}
$$

In the above, $\mathcal{T}_{m}([a, c], \psi$ ) (with $m=g$ or $g g$ ) represents the sum of the modified service times, $\tilde{B}$, of the users that arrived in the interval $[a, c]: 1)$ arrivals at around $q$ (independent arrivals as explained in footnote 10) occur for time period $|\psi(q,)$.$| as in$ subsection 2.4.2 and 2) if $\psi(q,$.$) is negative then the quantities added, \tilde{B}$, are negative of the service times, else $\tilde{B}$ are the service times themselves.

Let $\Theta:=\left(\Theta_{C}, \Theta_{U}\right)$. The function $\Theta: \Psi^{2} \rightarrow \Psi^{2}$ is affine linear (linear but for $|\mathcal{C}| \alpha^{-1}$ term). Let $\Theta_{\text {Lin }}$ represent the linear part. With this,

$$
\begin{aligned}
& \left\|\Theta\left(\psi_{c}, \psi_{u} ; \sigma\right)-\Theta\left(\psi_{c}^{\prime}, \psi_{u}^{\prime} ; \sigma\right)\right\|_{\infty, 2}=\left\|\Theta_{L i n}\left(\psi_{c}-\psi_{c}^{\prime}, \psi_{u}-\psi_{u}^{\prime}\right)\right\|_{\infty, 2} \\
& \leq \sqrt{\hat{B}}\left\|\psi_{u}-\psi_{u}^{\prime}\right\|_{\infty, 2}+\left\|\mathcal{T}_{g}\left(\left[0, \delta^{\sigma}(q)\right], \psi_{c}-\psi_{c}^{\prime}\right)\right\|_{\infty, 2} \\
& \quad+\left\|\mathcal{T}_{g g}\left(\left[0, \delta^{\sigma}(q)\right], \psi_{c}(|\mathcal{C}|)-\psi_{c}^{\prime}(|\mathcal{C}|)\right)\right\|_{\infty, 2} \\
& \quad+\frac{1}{\sqrt{\hat{B}}}\left(\left\|\mathcal{T}_{g}\left(\left[\delta^{\sigma}(q),|\mathcal{C}|\right], \psi_{c}-\psi_{c}^{\prime}\right)\right\|_{\infty, 2}+\left\|\mathcal{T}_{g g}\left(\left[\delta^{\sigma}(q),|\mathcal{C}|\right], \psi_{c}(|\mathcal{C}|)-\psi_{c}^{\prime}(|\mathcal{C}|)\right)\right\|_{\infty, 2}\right) .
\end{aligned}
$$

$12 C_{n}^{\sigma}$ is a sum of two monotone functions (recall from equation (15), $C_{n}(q)=T_{n}(q)+$ $\left[T_{n-1}|\mathcal{C}|-T_{n-1}(q)\right]$ ) while $U_{n}^{\sigma}$ is a monotone function of $q$ for all realizations, hence their moments will also have similar properties and hence both the moments are in $\mathcal{D}$. 
In the above, terms like $\left\|\mathcal{T}_{g}\left(\left[0, \delta^{\sigma}(q)\right], \psi_{c}\right)\right\|_{\infty, 2}$ imply the $\|.\|_{\infty, 2}$ norm of a $\psi \in \Psi$, where $\psi(q,$.$) represents the random workload, \mathcal{T}_{g}\left(\left[0, \delta^{\sigma}(q)\right], \psi_{c}\right)$. Note that for any type of arrivals (i.e., with $m=g$ or $m=g g$ ) and for any interval $I$ and time function $T$,

$$
\left|\mathcal{T}_{m}(I, T)\right| \leq \sum_{i} B_{i}
$$

where $B_{i}$ are the service times of the arrivals in the chosen time period $|T|$ and so it is clear that these type of terms to converges (in monotone fashion) to zero as $\hat{B} \rightarrow 0$. In fact even the terms like $1 / \sqrt{\hat{B}}\left|T_{m}(.,).\right|$ drop to zero as $\hat{B} \rightarrow 0$. Thus there exists a $\hat{\mathbf{B}}<\infty$ such that

$$
\left\|\Theta_{\text {Lin }}\right\|_{\infty, 2}<1 \text { for all } \hat{B}<\hat{\mathbf{B}} \text {. }
$$

Hence $\Theta$ is a contraction for all $\sigma$ and the contraction coefficient can also be chosen independent of $\sigma$, i.e., there exists $\hat{\mathbf{B}}$ and $L<1$ such that (for all $\hat{B}<\hat{\mathbf{B}}$ ),

$$
\left\|\Theta\left(\psi_{c}, \psi_{u} ; \sigma\right)-\Theta\left(\psi_{c}^{\prime}, \psi_{u}^{\prime} ; \sigma\right)\right\|_{\infty, 2} \leq L\left\|\left(\psi_{c}, \psi_{u}\right)-\left(\psi_{c}^{\prime}, \psi_{u}^{\prime}\right)\right\|_{\infty, 2} \text { for all } \sigma .
$$

Thus, from contraction mapping theorem, we have a unique fixed point $\left(C_{*}^{\sigma}, U_{*}^{\sigma}\right)$ for every $\sigma$ and these by construction of the map $\Theta$, given by equations (29)-(30), are the stationary quantities corresponding to $\left(C_{n}, U_{n}\right)$ with discretization levels $\sigma$.

Continuity of $\Theta$ with respect to $\sigma$ : From (31)-(32), difference $\Theta\left(C_{*}^{\sigma^{\prime}}, U_{*}^{\sigma^{\prime}} ; \sigma\right)-$ $\Theta\left(C_{*}^{\sigma^{\prime}}, U_{*}^{\sigma^{\prime}} ; \infty\right)$ is made up of terms like $\varpi$, the workload process defined for any $m=g$ or $g g$ and any $\psi \in \Psi$ by,

$$
\varpi_{m}^{\sigma}(q, . ; \psi):=\mathcal{T}_{m}\left(\left(\delta^{\sigma}(q), q\right], \psi\right) .
$$

Clearly $\varpi_{m}^{\sigma}\left(., . ; C_{*}^{\sigma^{\prime}}\right) \in \Psi$ for every $\sigma \leq \infty$ and for every $\sigma^{\prime}$ and using $^{13}$ (33)

$$
\left\|\varpi_{m}^{\sigma}\left(q, . ; C_{*}^{\sigma^{\prime}}\right)\right\|_{2} \leq 2 \hat{B}\left\|\mathcal{N}^{\sigma}(q, .)\right\|_{2}
$$

where $\mathcal{N}^{\sigma}(q,$.$) represents the number of customers that arrived in segment \left[\delta^{\sigma}(q), q\right]$ for a time duration of $C_{*}^{\sigma^{\prime}}(|\mathcal{C}|)$. Clearly ${ }^{14}$ using the properties of Poisson process,

$$
\begin{aligned}
\left\|\mathcal{N}^{\sigma}(q, .)\right\|_{2}^{2} & =E\left[\mathcal{N}^{\sigma}(q, .)^{2}\right] \\
& =\left(\lambda P_{Q}\left(\left[\delta^{\sigma}(q), q\right]\right) \| C_{*}^{\sigma^{\prime}}(|\mathcal{C}|)+\left(\lambda P_{Q}\left(\left(\delta^{\sigma}(q), q\right]\right) \| C_{*}^{\sigma^{\prime}}(|\mathcal{C}|)\right)^{2}\right) \\
& \leq\left(\lambda \frac{\left\|f_{Q}\right\|_{\infty}}{\sigma} C_{*}^{\sigma^{\prime}}(|\mathcal{C}|)+\left(\lambda \frac{\left\|f_{Q}\right\|_{\infty}}{\sigma} C_{*}^{\sigma^{\prime}}(|\mathcal{C}|)\right)^{2}\right) \text { for any } q .
\end{aligned}
$$

Hence for any given $\sigma^{\prime}$ and $m$,

$$
\lim _{\sigma \rightarrow \infty}\left\|\varpi_{m}^{\sigma}\left(., . ; C_{*}^{\sigma^{\prime}}\right)\right\|_{\infty, 2}=0 \quad \text { and similarly } \lim _{\sigma \rightarrow \infty}\left\|\varpi_{m}^{\sigma}\left(., . ; C_{*}^{\sigma^{\prime}}(|\mathcal{C}|)\right)\right\|_{\infty, 2}=0 .
$$

\footnotetext{
13 From equation (15), $C_{*}^{\sigma}(q)$ equals sum of stationary quantities corresponding to $T_{n}(q)$ and $T_{n-1}\left(|\mathcal{C}|-T_{n-1}(q)\right.$ and hence will be less than or equal to $2 C_{*}^{\sigma}(|\mathcal{C}|)$ in distribution and this is true for all $q$, i.e.,
}

$$
C_{*}^{\sigma}(q) \stackrel{\text { distribution }}{\leq} 2 C_{*}^{\sigma}(|\mathcal{C}|) \text { for all } q .
$$

Also, recall service times $B \leq \hat{B}$ with probability one.

14 because of independent arrivals as reasoned in footnotes 10 and 11 . 
From (31)-(32), difference

$$
\begin{aligned}
& \Theta_{C}\left(C_{*}^{\sigma^{\prime}}, U_{*}^{\sigma^{\prime}} ; \sigma\right)-\Theta_{C}\left(C_{*}^{\sigma^{\prime}}, U_{*}^{\sigma^{\prime}} ; \infty\right)=-\varpi_{g}^{\sigma}\left(., . ; C_{*}^{\sigma^{\prime}}\right)-\varpi_{g g}^{\sigma}\left(., . ; C_{*}^{\sigma^{\prime}}(|\mathcal{C}|)\right) \\
& \Theta_{U}\left(C_{*}^{\sigma^{\prime}}, U_{*}^{\sigma^{\prime}} ; \sigma\right)-\Theta_{U}\left(C_{*}^{\sigma^{\prime}}, U_{*}^{\sigma^{\prime}} ; \infty\right)=\frac{1}{\sqrt{\hat{B}}} \varpi_{g}^{\sigma}\left(., . ; C_{*}^{\sigma^{\prime}}\right)+\frac{1}{\sqrt{\hat{B}}} \varpi_{g g}^{\sigma}\left(., . ; C_{*}^{\sigma^{\prime}}(|\mathcal{C}|)\right)
\end{aligned}
$$

and thus for any $\sigma^{\prime} \leq \infty$,

$$
\left\|\Theta\left(C_{*}^{\sigma^{\prime}}, U_{*}^{\sigma^{\prime}} ; \sigma\right)-\Theta\left(C_{*}^{\sigma^{\prime}}, U_{*}^{\sigma^{\prime}} ; \infty\right)\right\|_{\infty, 2} \rightarrow 0 \text { as } \sigma \rightarrow \infty
$$

Continuity of fixed points with respect to $\sigma$ : By the definition of fixed points,

$$
\begin{aligned}
& \left\|\left(C_{*}^{\sigma}, U_{*}^{\sigma}\right)-\left(C_{*}^{\infty}, U_{*}^{\infty}\right)\right\|_{\infty, 2}=\left\|\Theta\left(C_{*}^{\sigma}, U_{*}^{\sigma} ; \sigma\right)-\Theta\left(C_{*}^{\infty}, U_{*}^{\infty} ; \infty\right)\right\|_{\infty, 2} \\
& \quad \leq\left\|\Theta\left(C_{*}^{\infty}, U_{*}^{\infty} ; \sigma\right)-\Theta\left(C_{*}^{\infty}, U_{*}^{\infty} ; \infty\right)\right\|_{\infty, 2}+\left\|\Theta\left(C_{*}^{\sigma}, U_{*}^{\sigma} ; \sigma\right)-\Theta\left(C_{*}^{\infty}, U_{*}^{\infty} ; \sigma\right)\right\|_{\infty, 2} \\
& \quad \leq\left\|\Theta\left(C_{*}^{\infty}, U_{*}^{\infty} ; \sigma\right)-\Theta\left(C_{*}^{\infty}, U_{*}^{\infty} ; \infty\right)\right\|_{\infty, 2}+L\left\|\left(C_{*}^{\sigma}, U_{*}^{\sigma}\right)-\left(C_{*}^{\infty}, U_{*}^{\infty}\right)\right\|_{\infty, 2} .
\end{aligned}
$$

And hence

$$
\left\|\left(C_{*}^{\sigma}, U_{*}^{\sigma}\right)-\left(C_{*}^{\infty}, U_{*}^{\infty}\right)\right\|_{\infty, 2} \leq \frac{1}{1-L}\left\|\Theta\left(C_{*}^{\infty}, U_{*}^{\infty} ; \sigma\right)-\Theta\left(C_{*}^{\infty}, U_{*}^{\infty} ; \infty\right)\right\|_{\infty, 2} .
$$

Thus, using (34), as $\sigma \rightarrow \infty$

$$
\left\|\left(C_{*}^{\sigma}, U_{*}^{\sigma}\right)-\left(C_{*}^{\infty}, U_{*}^{\infty}\right)\right\|_{\infty, 2} \rightarrow 0 .
$$

It is easy to check that $\left\|C_{*}^{\sigma}(q, .)\right\|_{2}=c_{*}^{2 \sigma}$. Thus, by the definition of $\|.\|_{\infty, 2}$ the theorem on second moments follows.

Lemma $3\left(\Psi,\|\cdot\|_{\infty, 2}\right)$ is a complete space.

Proof: Say $\psi_{n}$ is a Cauchy sequence. Then for any $q, \psi_{n}(q,$.$) is Cauchy in L^{2}$ space and hence there exist an $L^{2}$-limit say call it $\psi(q,$.$) . Now for any q$,

$$
\left\|\psi_{n}(q, .)-\psi(q, .)\right\|_{2}=\lim _{m \rightarrow \infty}\left\|\psi_{n}(q, .)-\psi_{m}(q, .)\right\|_{2}
$$

For any $\epsilon>0$ there exists $N$ such that

$$
\sup _{q}\left\|\psi_{m}(q, .)-\psi_{n}(q, .)\right\|_{2} \leq \epsilon \text { for all } n, m>N
$$

Combining the above two equations, whenever $n>N$

$$
\sup _{q}\left\|\psi_{n}(q, .)-\psi(q, .)\right\|_{2} \leq \epsilon
$$

and hence $\left\|\psi_{n}-\psi\right\|_{\infty, 2} \rightarrow 0$ as $n \rightarrow \infty$. $\diamond$

By completeness of $\mathcal{D}$ the map (of limits of $L^{2}$ norms) $q \rightarrow\|\psi(q, .)\|_{2}$ resides in $\mathcal{D}$. 


\section{Appendix C : Pareto Optimality}

Let $W$ be the set of vectors of expected waiting times achieved by a class of design policies. A vector $w_{1} \in W$ obtained by some policy dominates another vector $w_{2}$ if all entries of $w_{1}$ are smaller than or equal to those of $w_{2}$ with at least one entry being strictly smaller. This definition is given when $w_{1}$ has finite number of entries. We now extend the definition in an obvious way to our case, i.e., when $w_{1}$ is a function on $[0,|\mathcal{C}|]$ that is integrable with respect to Lebesgue measure, $\mathcal{L}$. A function $w_{1}$ is said to dominate another function $w_{2}$ if $w_{1} \leq w_{2}$ almost surely (w.r.t. Lebesgue measure) and if there exists a set $\mathcal{B}$ such that $w_{1}<w_{2}$ on $\mathcal{B}$ and $\mathcal{L}(\mathcal{B})>0$. A minimum vector in $W$ in the Pareto sense is one that is not dominated by any other vector in $W$.

Lemma 4 Let $\zeta$ be an integrable function on $[0,|\mathcal{C}|]$ with $\zeta>c$ almost surely, with $c \geq 0$. Let,

$$
w^{*}=\arg \min _{w \in W} \int_{0}^{|\mathcal{C}|} w(q) \zeta(q) d q,
$$

be a minimizer of the integral. Then $w^{*}$ is a Pareto optimal solution.

Proof : If it is not and there exists a vector $w_{1}$ which dominates $w^{*}$. Say $w_{1}<w^{*}$ on $\mathcal{B}$ with $\mathcal{L}(\mathcal{B})>0$ while $w_{1}=w^{*}$ on the complement, $\mathcal{B}^{c}$. Then

$\int_{0}^{|\mathcal{C}|}\left(w_{1}(q)-w^{*}(q)\right) \zeta(q) d q=\int_{\mathcal{B}}\left(w_{1}(q)-w^{*}(q)\right) \zeta(q) d q \leq-c \int_{\mathcal{B}}\left(w^{*}(q)-w_{1}(q)\right) d q<0$.

and so the optimality of $w^{*}$ is contradicted when $c>0$. When $c=0$, by definition of infimum one can find a subset of $\mathcal{B}$ on which $\zeta>\tilde{c}>0$ and the proof follows.

\section{References}

1. Francois Baccelli and Pierre Brémaud "Elements of Queueing Theory", volume 26. Applications of Mathematics, Springer-Verlag, 1991.

2. O.J. Boxma, J. Bruin and B. Fralix "Waiting times in polling systems with various service disciplines", EURANDOM report, June 2008.

3. O. Boxma "Workloads and waiting times in single-server systems with multiple user classes", Queuing Systems, 5, 185-214, 1989.

4. O.J. Boxma, W.P. Groenendijk, "Pseudo-Conservation Laws in Cyclic-Service Systems", Journal of Applied Probability, Vol. 24, No. 4, Dec 1987, 949-964.

5. O. J Boxma, H. Levy, U Yechiali, "Cyclic Reservation schemes for efficient operation of multiple-queue single-server systems", Annals of Operations Research, 1992, 187-208.

6. M. Sidi, H. Levy and S. W. Fuhrmann, "A queuing network with a single cyclically roving server", Queuing Systems 11, (special issue on Polling Models, Eds. H. Takagi and O. Boxma), pp.121-144, 1992.

7. J. E.G. Coffman and E. Gilbert. "A continuous polling system with constant service times", IEEE Trans. Inform. Theory 32 584-591, 1986.

8. J. E.G. Coffman and E. Gilbert. "Polling and greedy servers on the line", Queueing Systems 2115-145, 1987.

9. I. Eliazar. "The snowblower problem", Queueing Systems, 45, 357-380, 2003.

10. I. Eliazar. "From polling to snowplowing", Queueing Systems, 51(1-2), 115-133, 2005.

11. S. Fuhrmann and R. Copper. "Applications of the decomposition principle in $\mathrm{m} / \mathrm{g} / 1$ vacation models to two continuum cyclic queueing models", ATET Tech. J. 64 1091-1098, 1985.

12. L. Georgiadis and W. Szpankowski. "Stability of token passing ring", Queuing Syst., vol. 11, no. 1-2, pp. 7-33, 1992. 
13. D. Kroese and V. Schmidt. Queueing systems on the circle. Z. Oper. Res. 37(3) 303-331, 1993.

14. D. Kroese and V. Schmidt. Single-server queues with spatially distributed arrivals. Queueing Systems, 17, 317-345, 1994.

15. D. Kroese and V. Schmidt. Light-traffic analysis for queues with spatially distributed arrivals. Math. Oper. Research, 21, 135-157, 1996.

16. D. Kroese and V. Schmidt. A continuous polling system with general service times. The Annals of Applied Probability, Vol. 2, No. 4, pp. 906-927, Nov., 1992.

17. H. Takagi, "Analysis of Polling Systems", The MIT Press, 1986.

18. Foss, S.G. and Chernova, N.I., "Comparison Theorems and Ergodic Properties of the Polling Systems," Probl. Peredachi Inf., 1996, no. 4, pp. 46-71.

19. E. Altman, A. Khamisy and U. Yechiali , "On Elevator Polling with Globally Gated Regime", Queuing Systems, Vol. 11, (special issue on "Polling Models", Eds. H. Takagi and O. Boxma), pp. 85-90, 1992.

20. E. Altman, P. Konstantopoulos, and Z. Liu, "Stability, Monotonicity and Invariant Quantities in General Polling Systems", Queuing Syst., 1992, vol. 11, no. 12, pp. 3557.

21. E. Altman and H. Levy, "Queueing in space", Advances of Applied Probability 26, pp. 1095-1116, 1994.

22. A. Khamisy, E. Altman and M. Sidi , "Polling Systems with Synchronization Constraints", Annals of Operations Research, Vol. 35, special issue on Stochastic Modeling of Telecommunication Systems, Eds. P. Nain and K. W. Ross, pp. 231-267, 1992.

23. Umassdieselnet. http://prisms.cs.umass.edu/diesel/.

24. W. Saad, Z. Han, T. Basar, M. Debbah and A. Hjorungnes, "A Selfish Approach to Coalition Formation among Unmanned Air Vehicles in Wireless Networks", Gamenets, Istanbul, Turkey, 2009.

25. Saleh Yousefi, Eitan Altman, Rachid El-Azouzi and Mahmood Fathy, "Connectivity in vehicular ad hoc networks in presence of wireless mobile base-stations", in the Proceedings of the 7th International Conference on ITS Telecommunications, June 6-8, Sophia-Antipolis, France.

26. Y. Shi and Y. T. Hou, "Theoretical results on base station movement problem for sensor network," in IEEE INFOCOM 08, 2008.

27. M. M. B. Tariq, M. Ammar, and E. Zegura, "Message ferry route design for sparse ad hoc networks with mobile nodes", in Proc. of ACM MobiHoc, Florence, Italy, May 22-25, 2006, pp. 37-48.

28. V. Kavitha and E. Altman. "Continuous Polling with Rerouting and Applications to Ferry Assisted Wireless LANs" submitted to ValueTools 2011.

29. V. Kavitha and E. Altman. Analysis and design of message ferry routes in sensor networks using polling models. WiOpt May 31-Jun 04, Avignon, France, 2010.

30. Veeraruna Kavitha and Eitan Altman, "Queueing in Space: design of Message Ferry Routes in static adhoc networks", 21st International Teletraffic Congress (ITC 21) September 15-17, 2009, Paris, France

31. W. Whitt. A review of $l=\lambda w$ and extensions. Queueing Systems: Theory and Applications, Volume 9 Issue 3, Oct. 1991.

32. M. S. Berger. Nonlinearity and Functional Analysis. Academic Press, New York, 1977.

33. BV Limaye, "Functional Analysis", Wiley Eastern Ltd, New Delhi, India, 1981. 